\title{
THE DEGENERATE ANALOGUE OF ARIKI'S CATEGORIFICATION THEOREM
}

\author{
JONATHAN BRUNDAN AND ALEXANDER KLESHCHEV
}

\begin{abstract}
We explain how to deduce the degenerate analogue of Ariki's categorification theorem over the ground field $\mathbb{C}$ as an application of SchurWeyl duality for higher levels and the Kazhdan-Lusztig conjecture in finite type A. We also discuss some supplementary topics, including Young modules, tensoring with sign, tilting modules and Ringel duality.
\end{abstract}

\section{INTRODUCTION}

Ariki's categorification theorem proved in A1 is concerned with the representation theory of cyclotomic Hecke algebras of type $G(l, 1, d)$. For $l=1$, the result was conjectured earlier by Lascoux, Leclerc and Thibon [LLT], and a proof was also announced (but never published) by Grojnowski following G1.

For $d \geq 0$ and a dominant integral weight $\Lambda$ of level $l$ for the affine Lie algebra $\widehat{\mathfrak{s l}}_{e}(\mathbb{C})$, there is an associated cyclotomic Hecke algebra $\mathscr{H}_{d}^{\Lambda}$, namely, the quotient of the affine Hecke algebra $\mathscr{H}_{d}$ associated to the general linear group $G L_{d}$ by the two-sided ideal generated by the element

$$
\left(X_{1}-\xi^{m_{1}}\right) \cdots\left(X_{1}-\xi^{m_{l}}\right) \text {. }
$$

Here, the defining parameter $\xi$ of the Hecke algebra $\mathscr{H}_{d}$ should be taken to be a primitive complex eth root of unity, $m_{1}, \ldots, m_{l} \in \mathbb{Z} / e \mathbb{Z}$ are defined by expanding $\Lambda=\Lambda_{m_{1}}+\cdots+\Lambda_{m_{l}}$ in terms of fundamental dominant weights, and we are using the Bernstein presentation for $\mathscr{H}_{d}$.

The categorification theorem asserts that the direct sum

$$
\bigoplus_{d \geq 0}\left[\operatorname{Proj}\left(\mathscr{H}_{d}^{\Lambda}\right)\right]
$$

of the Grothendieck groups of the categories of finitely generated projective modules over these algebras can be identified with the standard $\mathbb{Z}$-form $V(\Lambda)_{\mathbb{Z}}$ for the irreducible highest weight $\widehat{\mathfrak{s l}}_{e}(\mathbb{C})$-module of highest weight $\Lambda$. Under the identification, the basis for the Grothendieck group arising from projective indecomposable modules corresponds to Lusztig's canonical basis, and the actions of the Chevalley generators of $\widehat{\mathfrak{s l}}_{e}(\mathbb{C})$ correspond to certain $i$-induction and $i$-restriction functors. Moreover, there is another natural family of $\mathscr{H}_{d}^{\Lambda}$ modules, the so-called Specht modules, such that the decomposition matrix describing composition multiplicities of Specht modules is the transpose of the matrix describing the expansion of the canonical basis for $V(\Lambda)_{\mathbb{Z}}$ in terms of the

1991 Mathematics Subject Classification. 17B20.

Key words and phrases. Categorification, cyclotomic Hecke algebra, parabolic category $\mathcal{O}$. Research supported in part by NSF grant no. DMS-0654147. 
monomial basis of an appropriate level $l$ Fock space $F(\Lambda)_{\mathbb{Z}}$ containing $V(\Lambda)_{\mathbb{Z}}$ as a submodule.

All of this also makes sense if $\xi$ is generic (not a root of unity), replacing $\widehat{\mathfrak{s l}}_{e}(\mathbb{C})$ with $\mathfrak{g l}_{\infty}(\mathbb{C})$ so that $m_{1}, \ldots, m_{l}$ are ordinary integers rather than integers modulo $e$. Even in the generic case, the proof is highly non-trivial (in levels bigger than one), depending ultimately on the $p$-adic analogue of the KazhdanLusztig conjecture formulated in [Z1], which is a special case of the DeligneLanglands conjecture proved in [KL2] (see also [CG, Chapter 8]), as well as Lusztig's geometric construction of canonical bases from [L3].

This article is concerned with the analogue of Ariki's categorification theorem for the degenerate cyclotomic Hecke algebra $H_{d}^{\Lambda}$, namely, the quotient of the degenerate affine Hecke algebra $H_{d}$ from [D] by the two-sided ideal generated by the element

$$
\left(x_{1}-m_{1}\right) \cdots\left(x_{1}-m_{l}\right) .
$$

Here, $m_{1}, \ldots, m_{l} \in \mathbb{Z}$ arise as before by writing the dominant integral weight $\Lambda$ for $\mathfrak{g l}_{\infty}(\mathbb{C})$ as $\Lambda=\Lambda_{m_{1}}+\cdots+\Lambda_{m_{l}}$. Remarkably, by [BK4, Corollary 2], the degenerate cyclotomic Hecke algebra $H_{d}^{\Lambda}$ is isomorphic to the non-degenerate cyclotomic Hecke algebra $\mathscr{H}_{d}^{\Lambda}$ for generic $\xi$. Combining this isomorphism with the cellular algebra structure on $H_{d}^{\Lambda}$ from [AMR, §6], which provides an intrinsic notion of Specht module in the degenerate setting, the degenerate analogue of Ariki's categorification theorem follows almost at once from Ariki's theorem for generic $\xi$ (and vice versa). It should also be possible to give a direct proof of the degenerate analogue without appealing to the isomorphism $H_{d}^{\Lambda} \cong \mathscr{H}_{d}^{\Lambda}$ by using Lusztig's geometric approach to the representation theory of $H_{d}$ from [L1, L5] to obtain the key multiplicity formula for standard modules in terms of intersection cohomology.

The goal in the remainder of the article is to explain a completely different way to prove the degenerate analogue of Ariki's categorification theorem, based instead on the Schur-Weyl duality for higher levels developed in BK3]. This duality is a generalization of classical Schur-Weyl duality, in which the group algebra of the symmetric group gets replaced by the degenerate cyclotomic Hecke algebra $H_{d}^{\Lambda}$, and the category of polynomial representations of the general linear group gets is replaced by certain blocks of a parabolic analogue of the Bernstein-Gelfand-Gelfand category $\mathcal{O}$ from $\mathrm{BGG}$. A great deal is known about parabolic category $\mathcal{O}$, thanks in part to the Kazhdan-Lusztig conjecture formulated in [KL1] that was proved in [BB, BrK]. Pushing this detailed information through Schur-Weyl duality, we recover in a very tidy way almost all known results about the representation theory of the degenerate cyclotomic Hecke algebras over the ground field $\mathbb{C}$, including the desired categorification theorem.

This means that there are two quite different ways to describe composition multiplicities of Specht modules in the degenerate case, one in terms of intersection cohomology of closures of nilpotent orbits of linear quivers, and the other in terms of parabolic Kazhdan-Lusztig polynomials associated to the symmetric group. The same coincidence has been observed before at the affine level: in AS Arakawa and Suzuki explained how to express the multiplicities of standard modules of degenerate affine Hecke algebras in terms of finite type 
A Kazhdan-Lusztig polynomials by means of another variation on Schur-Weyl duality involving category $\mathcal{O}$. The geometric explanation behind all these coincidences comes from a classic result of Zelevinsky [Z2]; we refer the reader to a recent paper of Henderson $[\mathrm{H}]$ for a comprehensive account (and extensions to the root of unity case).

In the Schur-Weyl duality approach, parabolic category $\mathcal{O}$ fits in very nicely into the categorification picture: its Grothendieck group plays the role of the level $l$ Fock space $F(\Lambda)_{\mathbb{Z}}$ mentioned above and the embedding of $V(\Lambda)_{\mathbb{Z}}$ into $F(\Lambda)_{\mathbb{Z}}$ corresponds at the level of categories to a "Schur functor" which maps projective $H_{d}^{\Lambda}$-modules to projective modules in parabolic category $\mathcal{O}$. This can be regarded as the extension to higher levels in the degenerate case of a conjecture of Leclerc and Thibon [LT], which was proved in level one in [VV].

There is actually a whole family of Schur-Weyl dualities relating various different type A parabolic category $\mathcal{O}$ 's to the Hecke algebra $H_{d}^{\Lambda}$, one for each permutation of the sequence $m_{1}, \ldots, m_{l}$ (corresponding to non-conjugate parabolic subalgebras with conjugate Levi factors). We usually consider only the standard one in which $m_{1} \geq \cdots \geq m_{l}$, as this leads to the most familiar combinatorics. In the other cases the parabolic category $\mathcal{O}$ that appears is usually not equivalent to the standard one, but it is always derived equivalent. This leads to some interesting twisted versions of the theory. We have included in the article some discussion of the situation for the opposite parabolic in which $m_{1} \leq \cdots \leq m_{l}$. This is particularly interesting as it explains the role of tilting modules and Ringel duality in the standard picture, leading to what can be interpreted as the degenerate analogue of the results of Mathas from [M].

The rest of the article is organized as follows.

- In section 2 we give a detailed account of the construction of the standard monomial, canonical and dual-canonical bases of $V(\Lambda)$ and $F(\Lambda)$. We also introduce a fourth basis for $F(\Lambda)$ which we call the quasicanonical basis, which has similar properties to the canonical basis but is not invariant under the bar involution. In the categorification picture the quasi-canonical basis corresponds to indecomposable projectives, whereas the canonical basis corresponds to tilting modules.

- In section 3 we derive the main categorification theorem (Theorem 3.10) which relates the three bases for $V(\Lambda)$ just mentioned to Specht modules, projective indecomposable modules and irreducible modules, respectively. The proof of this is a straightforward application of SchurWeyl duality for higher levels, the starting point being the analogous (known) categorification theorem relating $F(\Lambda)$ to parabolic category $\mathcal{O}$, which is a consequence of the Kazhdan-Lusztig conjecture.

- In section 4 we introduce Young modules and signed Young modules, and discuss their relationship with tilting modules and Ringel duality.

\section{Combinatorics of CANONicAl BASES}

In this section, we set up some basic notation related to the general linear Lie algebra and its quantized enveloping algebra $U$, in both finite and infinite rank. Then we fix a dominant weight $\Lambda$ and recall a standard construction of 
the irreducible $U$-module $V(\Lambda)$ of highest weight $\Lambda$, the main point being to explain in detail how various natural bases fit in with this construction.

2.1. Some combinatorics. Let $I$ be a non-empty set of consecutive integers and set $I_{+}:=I \cup(I+1)$. Let $P:=\bigoplus_{i \in I_{+}} \mathbb{Z} \Lambda_{i}$ be the weight lattice associated to the general linear Lie algebra $\mathfrak{g l}_{I_{+}}(\mathbb{C})$ of $I_{+} \times I_{+}$matrices; we refer to $\Lambda_{i}$ here as the $i$ th fundamental weight. If $I$ is bounded-below then $P$ can also be written as $P=\bigoplus_{i \in I_{+}} \mathbb{Z} \delta_{i}$ where the weights $\delta_{i}$ and $\Lambda_{j}$ are related by the formula

$$
\Lambda_{j}=\sum_{j \geq i \in I_{+}} \delta_{i}
$$

We give meaning to this formula when $I$ is not bounded-below by embedding $P$ into a larger space $\widehat{P}$ consisting of all formal $\mathbb{Z}$-linear combinations $\sum_{i \in I_{+}} a_{i} \delta_{i}$ such that $a_{i}=0$ for all sufficiently large $i$. Let $Q \subset P$ be the root lattice generated by the simple roots $\left\{\alpha_{i} \mid i \in I\right\}$ which are defined from

$$
\alpha_{i}=\delta_{i}-\delta_{i+1} .
$$

There is a canonical pairing (.,.) $: P \times Q \rightarrow \mathbb{Z}$ with $\left(\Lambda_{i}, \alpha_{j}\right)=\delta_{i, j}$ for $i \in I_{+}, j \in$ $I$. If $I$ is bounded-below this pairing is the restriction of the symmetric bilinear form on $P$ with respect to which the $\delta_{i}$ 's form an orthonormal basis.

Set $Q_{+}:=\sum_{i \in I} \mathbb{Z}_{\geq 0} \alpha_{i}$ and $P_{+}:=\sum_{i \in I_{+}} \mathbb{Z}_{\geq 0} \Lambda_{i}$. Let $h t(\alpha)$ denote the usual height of $\alpha \in Q_{+}$, i.e. the sum of its coefficients when expressed as a linear combination of simple roots. We will often need to work with $d$-tuples $\boldsymbol{i}=\left(i_{1}, \ldots, i_{d}\right) \in I^{d}$. The symmetric group $S_{d}$ acts naturally on $I^{d}$ by place permutation, and the orbits of $S_{d}$ on $I^{d}$ are the sets

$$
I^{\alpha}:=\left\{\boldsymbol{i} \in I^{d} \mid \alpha_{i_{1}}+\cdots+\alpha_{i_{d}}=\alpha\right\}
$$

parametrized by all $\alpha \in Q_{+}$of height $d$.

Suppose we are given a dominant weight $\Lambda \in P_{+}$. We can write it uniquely as

$$
\Lambda=\Lambda_{m_{1}}+\cdots+\Lambda_{m_{l}}
$$

for some $l \geq 0$ and $m_{1} \geq \cdots \geq m_{l}$. We refer to $l$ here as the level. We often identify $\Lambda$ with its diagram, namely, the array of boxes with rows indexed by $I_{+}$in increasing order from bottom to top, columns indexed by $1, \ldots, l$ from left to right, and a box in row $i$ and column $j$ whenever $i \leq m_{j}$. This definition makes sense even if the index set is not bounded-below, but in that case the diagram goes down forever. For example, taking $I=\mathbb{Z}$, the diagram of $\Lambda=\Lambda_{2}+\Lambda_{2}+\Lambda_{1}+\Lambda_{-1}$ is

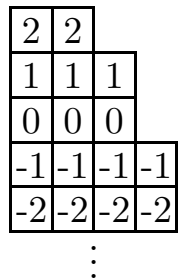

Here we have labelled all boxes on the $i$ th row by $i$.

A $\Lambda$-tableau is a diagram $A$ obtained by writing integers into the boxes of the diagram $\Lambda$. If $A$ is any $\Lambda$-tableau, we let $A(i, j)$ denote the entry in the $i$ th 
row and $j$ th column of $A$. We say $A$ is column-strict if its entries belong to $I_{+}$, they are strictly increasing from bottom to top in each column, and moreover all entries in the $i$ th row are equal to $i$ for all but finitely many rows (the final condition being vacuous if $I$ is bounded-below). We say $A$ is standard if it is column-strict and in addition its entries are weakly increasing from left to right in each row. Let $\mathrm{Col}^{\Lambda}$ (resp. $\mathrm{Std}^{\Lambda}$ ) denote the set of all column-strict (resp. standard) $\Lambda$-tableaux.

For any column-strict $\Lambda$-tableau $A$ and $1 \leq j \leq l$, define

$$
\begin{aligned}
\mathrm{wt}_{j}(A) & :=\sum_{m_{j} \geq i \in I_{+}} \delta_{A(i, j)}=\Lambda_{m_{j}}-\sum_{m_{j} \geq i \in I}\left(\alpha_{i}+\alpha_{i+1}+\cdots+\alpha_{A(i, j)-1}\right), \\
\operatorname{wt}(A) & :=\mathrm{wt}_{1}(A)+\cdots+\mathrm{wt}_{l}(A),
\end{aligned}
$$

the weight of the $j$ th column of $A$, and the weight of $A$, respectively. For any $\alpha \in Q_{+}$, let $\operatorname{Col}_{\alpha}^{\Lambda}\left(\operatorname{resp} . \operatorname{Std}_{\alpha}^{\Lambda}\right)$ denote the set of all column-strict (resp. standard) $\Lambda$-tableaux of weight $\Lambda-\alpha$. There is a unique column-strict $\Lambda$-tableau of weight $\Lambda$, namely, the ground-state tableau $A^{\Lambda}$ which has all entries in its $i$ th row equal to $i$ for all $i \in I_{+}$.

We also need the Bruhat order on $\mathrm{Col}^{\Lambda}$. This is defined by $A \leq B$ if $\operatorname{wt}(A)=$ $\mathrm{wt}(B)$ and

$$
\mathrm{wt}_{1}(A)+\cdots+\mathrm{wt}_{j}(A) \geq \mathrm{wt}_{1}(B)+\cdots+\mathrm{wt}_{j}(B)
$$

in the dominance ordering on $P$ for each $j=1, \ldots, l-1$. The Bruhat order has the basic property that $A<B$ if $B$ is obtained from $A$ by swapping entries $a<b$ in columns $i<j$.

2.2. The standard monomial basis. Let $U$ be the generic quantized enveloping algebra associated to $\mathfrak{g l}_{I_{+}}(\mathbb{C})$. Thus $U$ is the associative algebra over the field of rational functions $\mathbb{Q}(q)$ in an indeterminate $q$, with generators

$$
\left\{D_{i}, D_{i}^{-1} \mid i \in I_{+}\right\} \cup\left\{E_{i}, F_{i} \mid i \in I\right\}
$$

subject to the following well known relations:

$$
\begin{aligned}
& D_{i} D_{i}^{-1}=D_{i}^{-1} D_{i}=1, \quad E_{i} E_{j}=E_{j} E_{i} \quad \text { if }|i-j|>1, \\
& D_{i} D_{j}=D_{j} D_{i}, \quad E_{i}^{2} E_{j}+E_{j} E_{i}^{2}=\left(q+q^{-1}\right) E_{i} E_{j} E_{i} \quad \text { if }|i-j|=1, \\
& D_{i} E_{j} D_{i}^{-1}=q^{\left(\delta_{i}, \alpha_{j}\right)} E_{j}, \quad F_{i} F_{j}=F_{j} F_{i} \quad \text { if }|i-j|>1 \text {, } \\
& D_{i} F_{j} D_{i}^{-1}=q^{-\left(\delta_{i}, \alpha_{j}\right)} F_{j}, \quad F_{i}^{2} F_{j}+F_{j} F_{i}^{2}=\left(q+q^{-1}\right) F_{i} F_{j} F_{i} \quad \text { if }|i-j|=1 \text {, } \\
& E_{i} F_{j}-F_{j} E_{i}=\delta_{i, j} \frac{D_{i} D_{i+1}^{-1}-D_{i+1} D_{i}^{-1}}{q-q^{-1}} .
\end{aligned}
$$

We view $U$ as a Hopf algebra with comultiplication $\Delta$ defined on generators by

$$
\begin{aligned}
\Delta\left(D_{i}^{ \pm 1}\right) & =D_{i}^{ \pm 1} \otimes D_{i}^{ \pm 1}, \\
\Delta\left(E_{i}\right) & =1 \otimes E_{i}+E_{i} \otimes D_{i} D_{i+1}^{-1}, \\
\Delta\left(F_{i}\right) & =F_{i} \otimes 1+D_{i}^{-1} D_{i+1} \otimes F_{i} .
\end{aligned}
$$

For $\Lambda \in P_{+}$, let $V(\Lambda)$ denote the irreducible $U$-module of highest weight $\Lambda$, that is, the (unique up to isomorphism) irreducible $U$-module generated by a vector $v_{\Lambda}$ such that $E_{i} v_{\Lambda}=0$ for each $i \in I$ and $D_{i} v_{\Lambda}=q^{\left(\Lambda, \delta_{i}\right)} v_{\Lambda}$ for each $i \in I_{+}$. 
We are going to recall a well known direct construction of $V(\Lambda)$, beginning with the easiest case when $\Lambda$ is a fundamental weight.

Let $V$ denote the natural $U$-module with basis $\left\{v_{i} \mid i \in I_{+}\right\}$. The generators act on this basis by the rules

$$
D_{i} v_{j}=q^{\delta_{i, j}} v_{j}, \quad E_{i} v_{j}=\delta_{i+1, j} v_{i}, \quad F_{i} v_{j}=\delta_{i, j} v_{i+1} .
$$

Following [B1, §5] (noting the roles of $q$ and $q^{-1}$ are switched there), we define the $n$th quantum exterior power $\wedge^{n} V$ to be the $U$-submodule of $\bigotimes^{n} V$ spanned by the vectors

$$
v_{i_{1}} \wedge \cdots \wedge v_{i_{n}}:=\sum_{w \in S_{n}}(-q)^{\ell(w)} v_{i_{w(1)}} \otimes \cdots \otimes v_{i_{w(n)}}
$$

for all $i_{1}>\cdots>i_{n}$ from the index set $I_{+}$. Here, $\ell(w)$ denotes the usual length of a permutation $w \in S_{n}$.

If $I$ is bounded-below we have simply that

$$
V\left(\Lambda_{m}\right)=\bigwedge^{m+1-\inf (I)} V
$$

for each $m \in I_{+}$. The same thing is true if $\inf (I)=-\infty$ providing the right hand side of (2.5) is interpreted as the semi-infinite wedge $\bigwedge^{m+\infty} V$, that is, the $U$-module with basis consisting of all expressions of the form

$$
v_{i_{1}} \wedge v_{i_{2}} \wedge \cdots
$$

for $I_{+} \ni i_{1}>i_{2}>\cdots$ such that $i_{n}=m+1-n$ for $n \gg 0$.

More formally, $\bigwedge^{m+\infty} V$ is a direct limit of finite exterior powers. To write this down precisely, we need to let the index set $I$ vary: for any $k \in I$ let $I_{\geq k}:=\{i \in I \mid i \geq k\}$ and write $U_{\geq k}$ and $V_{\geq k}$ for the analogues of $U$ and $V$ defined with respect to the truncated index set $I_{\geq k}$. Obviously $U$ and $V$ are the unions of the corresponding truncated objects taken over all $k \in I$. Moreover for $k \leq m$ with $k-1 \in I$ there is a natural embedding of $U_{\geq k}$-modules

$$
\iota_{k}: \bigwedge^{m+1-k} V_{\geq k} \hookrightarrow \bigwedge^{m+2-k} V_{\geq(k-1)}
$$

sending $v_{i_{1}} \wedge \cdots \wedge v_{i_{m+1-k}}$ to $v_{i_{1}} \wedge \cdots \wedge v_{i_{m+1-k}} \wedge v_{k-1}$. When $I$ is not boundedbelow, the semi-infinite wedge $\bigwedge^{m+\infty} V$ is the direct limit of the finite exterior powers $\bigwedge^{m+1-k} V_{\geq k}$ over all $k \leq m$, taken with respect to the embeddings $\iota_{k}$. It is a $U_{\geq k}$-module for each $k$, hence actually a $U$-module.

Now suppose that $\Lambda \in P_{+}$is a dominant weight of arbitrary level and write it in the standard form (2.1). Set

$$
F(\Lambda):=V\left(\Lambda_{m_{1}}\right) \otimes \cdots \otimes V\left(\Lambda_{m_{l}}\right),
$$

a tensor product of $l$ fundamental representations. This $U$-module has an obvious monomial basis parametrized by the set $\mathrm{Col}^{\Lambda}$ of column-strict $\Lambda$-tableaux. More precisely, given $A \in \mathrm{Col}^{\Lambda}$, we set

$$
M_{A}:=\left(v_{A\left(m_{1}, 1\right)} \wedge v_{A\left(m_{1}-1,1\right)} \wedge \cdots\right) \otimes \cdots \otimes\left(v_{A\left(m_{l}, l\right)} \wedge v_{A\left(m_{l}-1, l\right)} \wedge \cdots\right),
$$

which is a tensor product of finite or semi-infinite wedges according to whether $I$ is bounded-below or not; informally, $M_{A}$ is the monomial in $F(\Lambda)$ obtained 
by reading the entries of $A$ down columns starting from the leftmost column. Then the monomial basis of $F(\Lambda)$ is the set

$$
\left\{M_{A} \mid A \in \mathrm{Col}^{\Lambda}\right\} \text {. }
$$

Each vector $M_{A}$ in this basis is of weight $\mathrm{wt}(A)$ as in (2.3), justifying that notation.

Since the $\Lambda$-weight space of $F(\Lambda)$ is one dimensional and all other weights are strictly smaller in the dominance order, $V(\Lambda)$ appears as a constituent of the integrable module $F(\Lambda)$ with multiplicity one. More precisely, the vector $v_{\Lambda}:=M_{A^{\Lambda}}$ is a canonical highest weight vector in $F(\Lambda)$ of weight $\Lambda$ and we can define $V(\Lambda)$ to be the $U$-submodule of $F(\Lambda)$ generated by this vector. In view of complete reducibility, there is also a canonical $U$-equivariant projection

$$
\pi: F(\Lambda) \rightarrow V(\Lambda) \text {. }
$$

For each $A \in \mathrm{Col}^{\Lambda}$, let $S_{A}:=\pi\left(M_{A}\right) \in V(\Lambda)$. These are the standard monomials in $V(\Lambda)$, and the standard monomial basis theorem asserts that vectors

$$
\left\{S_{A} \mid A \in \operatorname{Std}^{\Lambda}\right\}
$$

give a basis for $V(\Lambda)$. This is the quantum analogue of the classical standard monomial basis for the space of global sections of a line bundle on the flag variety, which goes back at least to Hodge. If $I$ is bounded-below the standard monomial basis theorem is proved (by no means for the first time!) in [B1, Theorem 26]. The standard monomial basis theorem when $I$ is not boundedbelow follows easily from the bounded-below case by taking direct limits, as we explain in the next paragraph.

Suppose then that $I$ is not bounded-below and take any $k \leq m_{l}$. Write $F(\Lambda)_{\geq k}$ and $V(\Lambda)_{\geq k}$ for the analogues of the modules $F(\Lambda)$ and $V(\Lambda)$ over the truncated algebra $U_{\geq k}$, i.e. working with the bounded-below index set $I_{\geq k}$. One checks easily that there is a commutative diagram

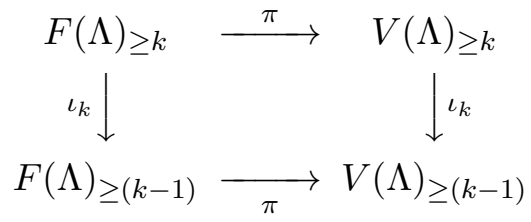

of $U_{\geq k}$-module homomorphisms. Here, the left hand vertical map is the tensor product of $l$ maps of the form (2.6), and we have that

$$
\iota_{k}\left(M_{A}\right)=M_{A \sqcup(k-1)^{l}},
$$

where $A \sqcup(k-1)^{l}$ denotes the tableau obtained obtained by adding an extra row of $l$ boxes, each labelled by $(k-1)$, to the bottom of $A$. The right hand vertical map is defined as the unique $U_{\geq k}$-module homomorphism mapping $v_{\Lambda} \in V(\Lambda)_{\geq k}$ to $v_{\Lambda} \in V(\Lambda)_{\geq(k-1)}$. The commutativity of the diagram implies that the right hand $\iota_{k}$ also has the property that

$$
\iota_{k}\left(S_{A}\right)=S_{A \sqcup(k-1)^{l}} .
$$

Moreover $F(\Lambda)$ and $V(\Lambda)$ are the direct limits of their truncated versions over all $k \leq m_{l}$, again taken with respect to the embeddings $\iota_{k}$. In view of (2.12), the 
monomial basis for $F(\Lambda)$ is the union of the monomial bases from all boundedbelow cases. Similarly by (2.13), the standard monomial basis for $V(\Lambda)$ is the union of the standard monomial bases from all $V(\Lambda)_{>k}$. This proves the standard monomial basis theorem for $V(\Lambda)$ in the case that $I$ is not boundedbelow.

2.3. The dual-canonical basis. In this subsection we are going to recall a slightly unorthodox definition of Lusztig's dual-canonical basis of $V(\Lambda)$ (which is the upper global crystal base of Kashiwara) following [B1, §7]. To get started, we need Lusztig's bar involution on $F(\Lambda)$. The bar involution on $U$ is the automorphism - : $U \rightarrow U$ that is anti-linear with respect to the field automorphism $\mathbb{Q}(q) \rightarrow \mathbb{Q}(q), f(q) \mapsto f\left(q^{-1}\right)$ and satisfies

$$
\overline{E_{i}}=E_{i}, \quad \overline{F_{i}}=F_{i}, \quad \overline{D_{i}}=D_{i}^{-1} .
$$

By a compatible bar involution on a $U$-module $M$ we mean an anti-linear involution $-: M \rightarrow M$ such that $\overline{u v}=\bar{u} \bar{v}$ for each $u \in U, v \in M$. The fundamental module $V\left(\Lambda_{m}\right)$ possesses a compatible bar involution which fixes each of its basis vectors of the form $v_{i_{1}} \wedge v_{i_{2}} \wedge \cdots$ for $i_{1}>i_{2}>\cdots$. From this and a general construction due to Lusztig involving the quasi- $R$-matrix [L4, §27.3], we get a compatible bar involution on the tensor product $F(\Lambda)$ from (2.7). It has the crucial property that

$$
\overline{M_{A}}=M_{A}+\left(\mathrm{a} \mathbb{Z}\left[q, q^{-1}\right] \text {-linear combination of } M_{B} \text { 's for } B<A\right)
$$

where $<$ is the Bruhat order on column-strict tableaux. This is explained in more detail in [B1, §5] in the case when $I$ is bounded-below. In view of the following lemma, the bar involution on $F(\Lambda)$ when $I$ is not bounded-below is the limit of the bar involutions on each of the truncations $F(\Lambda)_{\geq k}$.

Lemma 2.1. Suppose that $I$ is not bounded-below and take $k \leq m_{l}$. The bar involution commutes with the natural embedding $\iota_{k}: F(\Lambda)_{\geq k} \hookrightarrow F(\Lambda)_{\geq(k-1)}$.

Proof. This follows from the definition of the bar involution and the fact that the quasi- $R$-matrix attached to $U_{\geq(k-1)}$ is equal to the quasi- $R$-matrix attached to $U_{\geq k}$ plus a sum of terms which annihilate vectors in $\iota_{k}\left(F(\Lambda)_{\geq k}\right)$ by weight considerations.

Applying Lusztig's lemma [L4, Lemma 24.2.1], we can now define the dualcanonical basis

$$
\left\{L_{A} \mid A \in \mathrm{Col}^{\Lambda}\right\}
$$

of $F(\Lambda)$ by declaring that $L_{A}$ is the unique bar-invariant vector such that

$$
L_{A}=M_{A}+\left(\text { a } q \mathbb{Z}[q] \text {-linear combination of } M_{B} \text { 's for various } B<A\right) \text {. }
$$

Using Lemma 2.1, (2.12) and the definition, it is easy to check for any $k \leq m_{l}$ with $k-1 \in I$ that the embedding $\iota_{k}: F(\Lambda)_{\geq k} \hookrightarrow F(\Lambda)_{\geq(k-1)}$ satisfies

$$
\iota_{k}\left(L_{A}\right)=L_{A \sqcup(k-1)^{l}} .
$$

This means that, like the monomial basis, the dual-canonical basis for $F(\Lambda)$ in the case that $I$ is not bounded-below is the union of the dual-canonical bases from all bounded-below cases. 
The polynomials $d_{A, B}(q), p_{A, B}(q) \in \mathbb{Z}[q]$ defined from

$$
\begin{aligned}
M_{B} & =\sum_{A \in \mathrm{Col}^{\Lambda}} d_{A, B}(q) L_{A}, \\
L_{B} & =\sum_{A \in \mathrm{Col}^{\Lambda}} p_{A, B}(-q) M_{A}
\end{aligned}
$$

satisfy $p_{A, A}(q)=d_{A, A}(q)=1$ and $p_{A, B}(q)=d_{A, B}(q)=0$ unless $A \leq B$. In B1, Remark 14] one finds explicit formulae expressing these polynomials in terms of finite type A Kazhdan-Lusztig polynomials. Let us record the appropriate formla for the $p_{A, B}(q)$ 's, which are just Deodhar's parabolic Kazhdan-Lusztig polynomials for the symmetric group. It suffices in view of (2.12) and (2.15) to do this in the case that $I$ is bounded-below. Consider the natural right action of $S_{n}$ on $I_{+}^{n}$ by place permutation. For $A \in \mathrm{Col}^{\Lambda}$, let $\left(a_{1}, \ldots, a_{n}\right)$ be column-reading of $A$, that is, the tuple obtained by reading the entries of $A$ down columns starting with the leftmost column. Define $w_{A}$ to be the unique element of $S_{n}$ of minimal length such that $\left(a_{1}, \ldots, a_{n}\right) w_{A}$ is a weakly increasing sequence. Also let $Z_{A}$ be the stabilizer in $S_{n}$ of this weakly increasing sequence. Then for any $A \leq B$ we have that

$$
p_{A, B}(q)=q^{\ell\left(w_{B}\right)-\ell\left(w_{A}\right)} \sum_{z \in Z_{A}}(-1)^{\ell(z)} P_{w_{A} z, w_{B}}\left(q^{-2}\right),
$$

where $P_{x, y}(q)$ is the usual Kazhdan-Lusztig polynomial exactly as in [KL1]. The formula (2.18) should be compared with the second formula from [BGS, Theorem 3.11.4(iv)].

Now we pass from $F(\Lambda)$ to $V(\Lambda)$. The bar involution on $F(\Lambda)$ restricts to give a well-defined compatible bar involution on $V(\Lambda)$. It is the unique compatible bar involution on $V(\Lambda)$ fixing the highest weight vector $v_{\Lambda}$. For $A \in \operatorname{Std}^{\Lambda}$ define $D_{A}:=\pi\left(L_{A}\right) \in V(\Lambda)$, so that

$$
D_{B}=\sum_{A \in \mathrm{Col}^{\Lambda}} p_{A, B}(-q) S_{A} .
$$

In view of the following theorem, the vectors

$$
\left\{D_{A} \mid A \in \operatorname{Std}^{\Lambda}\right\}
$$

constitute the dual-canonical basis of $V(\Lambda)$. Moreover we have that

$$
S_{B}=\sum_{A \in \operatorname{Std}^{\Lambda}} d_{A, B}(q) D_{A}
$$

for $B \in \mathrm{Col}^{\Lambda}$. Finally by (2.15) and the commutativity of the diagram (2.11), we again get that

$$
\iota_{k}\left(D_{A}\right)=D_{A \sqcup(k-1)^{l}},
$$

i.e. the dual-canonical basis for $V(\Lambda)$ when $I$ is not bounded-below is the union of the dual-canonical bases from all the bounded-below cases.

Theorem 2.2. Each $D_{A}$ is bar-invariant and the vectors $\left\{D_{A} \mid A \in \operatorname{Std}^{\Lambda}\right\}$ form a basis for $V(\Lambda)$ which coincides with Lusztig's dual-canonical basis. Moreover, $\pi\left(L_{A}\right)=0$ if $A$ is not standard. 
Proof. It suffices to prove the theorem in the case that $I$ is bounded-below. In that case, everything follows from [B1, Theorem 26]. The fact that the $D_{A}$ 's coincide with the usual dual-canonical basis in the sense of Lusztig is explained in [B1, Remark 27].

Corollary 2.3. The vectors $\left\{L_{A} \mid A \in \mathrm{Col}^{\Lambda} \backslash \operatorname{Std}^{\Lambda}\right\}$ give a basis for $\operatorname{ker} \pi$.

2.4. The crystal graph. The actions of the Chevalley generators $E_{i}$ and $F_{i}$ on the dual-canonical bases of $F(\Lambda)$ and $V(\Lambda)$ are reflected by an underlying crystal graph. We wish to briefly recall the definition of this important combinatorial object.

Suppose we are given a column-strict tableau $A \in \mathrm{Col}^{\Lambda}$ and $i \in I$. Enumerate the boxes of $A$ that contain the entries $i$ or $(i+1)$ as $b_{1}, \ldots, b_{n}$ in column-reading order, i.e. working in order down columns starting with the leftmost column. Thus if $r<s$ then $b_{r}$ is either located in a column strictly to the left of $b_{s}$, or $b_{r}$ is in the same column as but strictly above $b_{s}$. Then we define the reduced $i$-signature $\left(\sigma_{1}, \ldots, \sigma_{n}\right)$ of $A$ by applying the following algorithm. Start with the sequence $\left(\sigma_{1}, \ldots, \sigma_{n}\right)$ in which $\sigma_{r}=+$ if $b_{r}$ contains the entry $i$ and $\sigma_{r}=-$ if $b_{r}$ contains the entry $(i+1)$. If we can find $1 \leq r<s \leq n$ such that $\sigma_{r}=-$, $\sigma_{s}=+$ and $\sigma_{r+1}=\cdots=\sigma_{s-1}=0$ then we replace $\sigma_{r}$ and $\sigma_{s}$ by 0 . Keep doing this until we are left with a sequence $\left(\sigma_{1}, \ldots, \sigma_{n}\right)$ in which no - appears to the left of a + . This is the reduced $i$-signature of $A$. Then define

$$
\begin{aligned}
\varepsilon_{i}(A) & :=\#\left\{r=1, \ldots, n \mid \sigma_{r}=-\right\}, \\
\varphi_{i}(A) & :=\#\left\{r=1, \ldots, n \mid \sigma_{r}=+\right\} .
\end{aligned}
$$

If $\varepsilon_{i}(A)=0$ then we set $\tilde{e}_{i}(A):=\odot$; otherwise we define $\tilde{e}_{i}(A)$ to be the columnstrict tableau obtained by replacing the entry $(i+1)$ in box $b_{r}$ by $i$, where $r$ indexes the leftmost - in the reduced $i$-signature. Similarly, if $\varphi_{i}(A)=0$ then we set $\tilde{f}_{i}(A):=\odot$; otherwise we define $\tilde{f}_{i}(A)$ to be the column-strict tableau obtained by replacing the $i$ in box $b_{r}$ by $(i+1)$, where $r$ indexes the rightmost + in the reduced $i$-signature. This defines the crystal operators

$$
\tilde{e}_{i}, \tilde{f}_{i}: \mathrm{Col}^{\Lambda} \rightarrow \mathrm{Col}^{\Lambda} \sqcup\{\odot\} .
$$

Moreover it is obviously the case that

$$
\left(\operatorname{wt}(A), \alpha_{i}\right)=\varphi_{i}(A)-\varepsilon_{i}(A) \text {. }
$$

The datum $\left(\mathrm{Col}^{\Lambda}, \tilde{e}_{i}, \tilde{f}_{i}, \varepsilon_{i}, \varphi_{i}\right.$,wt) just defined is the crystal in the sense of Kashiwara K2 associated to the module $F(\Lambda)$.

The crystal graph is then the colored, directed graph with vertex set $\mathrm{Col}^{\Lambda}$ and an edge $A \stackrel{i}{\rightarrow} B$ of color $i$ if $B=\tilde{f}_{i}(A)$; equivalently, $A=\tilde{e}_{i}(B)$. The connected component of this graph containing the ground-state tableau $A^{\Lambda}$ has vertex set $\operatorname{Std}^{\Lambda}$. This connected component is the crystal graph associated to the highest weight module $V(\Lambda)$; for $I$ bounded-below it is the same as the crystal graph from [KN]. 
Lemma 2.4. For $A \in \mathrm{Col}^{\Lambda}$ and $i \in I$ we have that

$$
\begin{gathered}
E_{i} L_{A}=\left[\varepsilon_{i}(A)\right] L_{\tilde{e}_{i}(A)}+\sum_{\substack{B \in \mathrm{Col}^{\Lambda} \\
\varepsilon_{i}(B)<\varepsilon_{i}(A)-1}} x_{A, B}^{i}(q) L_{B}, \\
F_{i} L_{A}=\left[\varphi_{i}(A)\right] L_{\tilde{f}_{i}(A)}+\sum_{\substack{B \in \operatorname{Col}^{\Lambda} \\
\varphi_{i}(B)<\varphi_{i}(A)-1}}^{i} y_{A, B}^{i}(q) L_{B},
\end{gathered}
$$

for bar-invariant $x_{A, B}^{i}(q) \in q^{\varepsilon_{i}(A)-2} \mathbb{Z}\left[q^{-1}\right]$ and $y_{A, B}^{i}(q) \in q^{\varphi_{i}(A)-2} \mathbb{Z}\left[q^{-1}\right]$. The analogous statement with $L_{A}$ replaced by $D_{A}$ and $\mathrm{Col}^{\Lambda}$ replaced by $\operatorname{Std}^{\Lambda}$ everywhere is also true.

Proof. Our dual-canonical basis is the upper global crystal base in the sense of Kashiwara associated to the tensor product (2.7), and the crystal structure defined above is precisely the underlying crystal by Kashiwara's tensor product rule for crystals. Given this, the first part of the lemma follows from [K1, Proposition 5.3.1]. The final statement then follows on applying the projection $\pi$, using the final statement of Theorem 2.2 .

2.5. A twisted version. We can modify the construction of $V(\Lambda)$ and its dualcanonical basis by changing the order of the tensor product of fundamental representations in the definition (2.7) of the module $F(\Lambda)$. As explained in detail in [B1, Theorem 26], this modification leads naturally to a family of monomial bases for $V(\Lambda)$, one for each permutation of the sequence $m_{1}, \ldots, m_{l}$, but always produces the same dual-canonical basis at the end. We want to briefly explain one of these twisted versions, namely, the one which is at the opposite extreme to the construction explained so far.

Continue with $\Lambda$ fixed as in (2.1), so that $m_{1} \geq \cdots \geq m_{l}$. Define

$$
\widetilde{F}(\Lambda):=V\left(\Lambda_{m_{l}}\right) \otimes \cdots \otimes V\left(\Lambda_{m_{1}}\right),
$$

so we have taken the tensor product in the reverse order to that of 92.2 . Let

$$
\widetilde{M}_{A}:=\left(v_{A\left(m_{l}, l\right)} \wedge v_{A\left(m_{l}-1, l\right)} \wedge \cdots\right) \otimes \cdots \otimes\left(v_{A\left(m_{1}, 1\right)} \wedge v_{A\left(m_{1}-1,1\right)} \wedge \cdots\right)
$$

denote the monomial obtained by reading the entries of $A$ down columns starting from the rightmost column; we refer to this way of reading the entries of $A$ as reverse-column-reading. This gives us the obvious monomial basis for this space:

$$
\left\{\widetilde{M}_{A} \mid A \in \mathrm{Col}^{\Lambda}\right\} \text {. }
$$

There is also a bar involution on $\widetilde{F}(\Lambda)$ defined exactly as before. From this, we get the dual-canonical basis

$$
\left\{\widetilde{L}_{A} \mid A \in \mathrm{Col}^{\Lambda}\right\}
$$

in which $\widetilde{L}_{A}$ is the unique bar-invariant vector such that

$$
\widetilde{L}_{A}=\widetilde{M}_{A}+\left(\text { a } q \mathbb{Z}[q] \text {-linear combination of } \widetilde{M}_{B} \text { 's for various } B>A\right) \text {. }
$$


Note the inequality $B>A$ here is the reverse of the analogous inequality in the definition of $L_{A}$ in 2.3 . We get a twisted version of the polynomials from (2.16) by expanding

$$
\widetilde{M}_{B}=\sum_{A \in \mathrm{Col}^{\Lambda}} \widetilde{d}_{A, B}(q) \widetilde{L}_{A}
$$

Thus $\widetilde{d}_{A, A}(q)=1$ and $\widetilde{d}_{A, B}(q)=0$ unless $A \geq B$.

The crystal graph in this setting is defined in a similar way to the crystal graph in 92.4 , but starting from the enumeration $b_{1}, \ldots, b_{n}$ of the boxes of $A$ containing the entries $i$ or $(i+1)$ in reverse-column-reading order, i.e. if $r<s$ then $b_{r}$ is either to the right of $b_{s}$ or it is in the same column but strictly above $b_{s}$. We call the resulting crystal structure on the set $\mathrm{Col}^{\Lambda}$ the reverse crystal structure. Let $\mathrm{Rev}^{\Lambda}$ denote the subset of $\mathrm{Col}^{\Lambda}$ that indexes the vertices from the connected component of the reverse crystal graph generated by the groundstate tableau $A^{\Lambda}$, and set $\operatorname{Rev}_{\alpha}^{\Lambda}:=\operatorname{Rev}^{\Lambda} \cap \operatorname{Col}_{\alpha}^{\Lambda}$. The set $\operatorname{Rev}^{\Lambda}$ can be described directly as the set of all reverse-standard $\Lambda$-tableaux, that is, the column-strict $\Lambda$-tableaux with the property that, on sliding all boxes in the $i$ th column up by $\left(m_{1}-m_{i}\right)$ places, the entries within each row are weakly decreasing from left to right; this combinatorial description can be derived from [B1, (2.2)].

There is a canonical crystal isomorphism

$$
\operatorname{Rev}^{\Lambda} \stackrel{\sim}{\rightarrow} \operatorname{Std}^{\Lambda}, \quad A \mapsto A^{\downarrow}
$$

between $\operatorname{Rev}^{\Lambda}$ equipped with the reverse crystal structure and $\operatorname{Std}^{\Lambda}$ equipped with the usual crystal structure. This map can easily be computed as a special case of the rectification map of Lascoux and Schützenberger [LS]; see also [F, $\S A .5]$ and [B1, (2.3)]. We recall this explicitly after the statement of Theorem 2.5 below.

Again we have a canonical projection

$$
\widetilde{\pi}: \widetilde{F}(\Lambda) \rightarrow V(\Lambda)
$$

mapping $\widetilde{M}_{A^{\Lambda}}$ to $v_{\Lambda}$. We set $\widetilde{S}_{A}:=\widetilde{\pi}\left(\widetilde{M}_{A}\right)$ for each $A \in \mathrm{Col}^{\Lambda}$ and $\widetilde{D}_{A}:=\widetilde{\pi}\left(\widetilde{L}_{A}\right)$ for $A \in \operatorname{Rev}^{\Lambda}$. For $I$ bounded-below the following theorem is another special case of [B1, Theorem 26]; it extends to arbitrary $I$ by the usual direct limit argument.

Theorem 2.5. The vectors $\left\{\widetilde{S}_{A} \mid A \in \operatorname{Rev}^{\Lambda}\right\}$ and $\left\{\widetilde{D}_{A} \mid A \in \operatorname{Rev}^{\Lambda}\right\}$ give bases for $V(\Lambda)$. Moreover for each $A \in \operatorname{Rev}^{\Lambda}$ we have that

$$
\widetilde{D}_{A}=D_{A \downarrow} \text {. }
$$

Hence the basis $\left\{\widetilde{D}_{A} \mid A \in \operatorname{Rev}^{\Lambda}\right\}$ coincides with Lusztig's dual-canonical basis (but it is parametrized in a non-standard way).

The rectification map (2.31) can be computed explicitly as follows.

Assume to start with that $I$ is bounded-below, and recall the notion of row insertion from [F, §1.1]. Given $A \in \operatorname{Rev}^{\Lambda}$, read the entries of $A$ in reversecolumn-reading order to obtain a sequence of integers $a_{1}, \ldots, a_{n}$. Then, starting from the diagram of $\Lambda$ with all boxes empty, we use row insertion to successively insert the entries $a_{1}, \ldots, a_{n}$ into the bottom row, bumping larger entries up and 
writing entries within each row in weakly increasing order, to obtain $A^{\downarrow}$ at the end. This means that at the $r$ th step the diagram has $(r-1)$ boxes filled in and we need to insert the entry $a_{r}$ into the bottom row. If $a_{r}$ is $\geq$ all entries in this row, simply add it to the first empty box in the row; else find the smallest entry $b$ in the row that is strictly larger than $a_{r}$, replace this entry $b$ by $a_{r}$, then insert $b$ into the next row up in a similar fashion.

To drop the assumption that $I$ is bounded-below, we just note that

$$
\left(A \sqcup(k-1)^{l}\right)^{\downarrow}=A^{\downarrow} \sqcup(k-1)^{l}
$$

if all entries of $A$ are $\geq k$. Hence $A^{\downarrow}$ when $I$ is not bounded-below can be computed by first choosing $k$ so that all entries in the $(k-1)$ th row of $A$ equal $k-1$, then applying the above algorithm to the bounded-below tableau in rows $k$ and above, leaving rows below the $k$ th row untouched.

Here are some examples of the map $A \mapsto A^{\downarrow}$ :

$$
\begin{aligned}
& \begin{array}{|l|l|}
\hline 5 & \\
\hline 4 & 2 \\
\hline 3 & 1 \\
\hline
\end{array} \mapsto \begin{array}{|l|l|}
\hline 5 & \\
\hline 2 & 4 \\
\hline 1 & 3 \\
\hline 1
\end{array} \quad \begin{array}{|l|l|}
\hline 5 & \\
\hline 4 & 3 \\
\hline 2 & 1 \\
\hline
\end{array} \mapsto \begin{array}{|l|l|}
\hline 5 & \\
\hline 3 & 4 \\
\hline 1 & 2 \\
\hline
\end{array} \quad \begin{array}{|l|l|l|}
\hline 5 & \\
\hline 3 & 4 \\
\hline 1 & 2 \\
\hline
\end{array} \mapsto \begin{array}{|l|l|}
\hline 4 & \\
\hline 2 & 5 \\
\hline 1 & 3 \\
\hline
\end{array} \\
& \begin{array}{|l|l|}
\hline 5 & \\
\hline 4 & 3 \\
\hline 1 & 2 \\
\hline
\end{array} \mapsto \begin{array}{|l|l|}
\hline 3 & \\
\hline 2 & 5 \\
\hline 1 & 4 \\
\hline
\end{array} \quad \begin{array}{|l|l|}
\hline 5 & \\
\hline 3 & 4 \\
\hline 2 & 1 \\
\hline
\end{array} \mapsto \begin{array}{|l|l|}
\hline 4 & \\
\hline 3 & 5 \\
\hline 1 & 2 \\
\hline
\end{array}
\end{aligned}
$$

As these examples may suggest, it is always the case that $A \geq A^{\downarrow}$ in the Bruhat order, as follows from Corollary 2.8 below.

The inverse of the map (2.31) gives another weight-preserving bijection

$$
\operatorname{Std}^{\Lambda} \stackrel{\sim}{\rightarrow} \operatorname{Rev}^{\Lambda}, \quad A \mapsto A^{\uparrow} .
$$

This can also be computed in terms of some row insertions. We explain just in the case that $I$ is bounded-below. Take $A \in \operatorname{Std}^{\Lambda}$ and read its entries $a_{1}, \ldots, a_{n}$ in column order. Start with the empty diagram obtained by sliding all boxes in the $i$ th column of the diagram of $\Lambda$ up by $\left(m_{1}-m_{i}\right)$ places. Then successively insert $a_{n}, \ldots, a_{1}$ into the top row of this diagram, this time bumping smaller entries down and writing entries within each row in weakly decreasing order. Thus, at the $r$ th step, we need to insert $a_{n+1-r}$ into the top row. If $a_{n+1-r}$ is $\leq$ all entries in this row, we simply add it to the first empty box in the row; else find the largest entry $b$ in the row that is strictly smaller than $a_{n+1-r}$, replace $b$ by $a_{n+1-r}$, then insert $b$ into the next row down in a similar fashion. When all insertions are done, we then slide all boxes in the $i$ th column back down by $\left(m_{1}-m_{i}\right)$ places to end up with the desired tableau $A^{\uparrow}$ of shape $\Lambda$. It is the case that $A^{\uparrow} \geq A$ in the Bruhat order.

2.6. The canonical and quasi-canonical bases. We are ready to define two more natural bases for $F(\Lambda)$. First, we have the canonical basis

$$
\left\{T_{A} \mid A \in \mathrm{Col}^{\Lambda}\right\}
$$


from [L4, §27.3]. By definition, $T_{A}$ is the unique bar-invariant vector in $F(\Lambda)$ such that

$$
T_{A}=M_{A}+\left(\mathrm{a} q^{-1} \mathbb{Z}\left[q^{-1}\right] \text {-linear combination of } M_{B} \text { 's for various } B<A\right) \text {. }
$$

Second, we have the quasi-canonical basis

$$
\left\{P_{A} \mid A \in \mathrm{Col}^{\Lambda}\right\}
$$

which is defined from the equations

$$
\begin{aligned}
P_{A} & =\sum_{B \in \mathrm{Col}^{\Lambda}} d_{A, B}(q) M_{B}, \\
M_{A} & =\sum_{B \in \mathrm{Col}^{\Lambda}} p_{A, B}(-q) P_{B} .
\end{aligned}
$$

(We have simply transposed the transition matrices from (2.16)-(2.17).)

The canonical and quasi-canonical bases have very similar properties, since both are dual to the dual-canonical basis under certain pairings. In the case of the canonical basis, there is a twist here since it is actually the dual basis to the dual-canonical basis on the space $\widetilde{F}(\Lambda)$ rather than on the space $F(\Lambda)$ itself. In this article, we usually prefer to work with the quasi-canonical basis rather than the canonical basis, in part to avoid this awkward twist but also because it is more convenient from the point of view of the categorifications studied later on.

The sense in which the quasi-canonical basis is dual to the dual-canonical basis is as follows. Introduce a sesquilinear form $\langle.,$.$\rangle on F(\Lambda)$ (anti-linear in the first argument, linear in the second) such that

$$
\left\langle M_{A}, \overline{M_{B}}\right\rangle=\delta_{A, B}
$$

for each $A, B \in \mathrm{Col}^{\Lambda}$. A straightforward computation using (2.36) and the formula obtained from (2.17) by applying the bar involution then shows that

$$
\left\langle P_{A}, L_{B}\right\rangle=\delta_{A, B}
$$

for $A, B \in \mathrm{Col}^{\Lambda}$. To formulate another property of the form $\langle.,$.$\rangle , let \tau: U \rightarrow U$ be the anti-linear anti-automorphism such that

$$
\tau\left(E_{i}\right)=q F_{i} D_{i}^{-1} D_{i+1}, \quad \tau\left(F_{i}\right)=q^{-1} D_{i} D_{i+1}^{-1} E_{i}, \quad \tau\left(D_{i}\right)=D_{i}^{-1} .
$$

Then we have that

$$
\langle u x, y\rangle=\langle x, \tau(u) y\rangle
$$

for $x, y \in F(\Lambda)$ and $u \in U$. This follows by a direct check using (2.38).

To make the sense in which the canonical basis is dual to the dual-canonical basis precise, recall the bases (2.28)-(2.29) for $\widetilde{F}(\Lambda)$. Define a bilinear pairing $(.,):. \widetilde{F}(\Lambda) \times F(\Lambda) \rightarrow \mathbb{Q}(q)$ by declaring that

$$
\left(\widetilde{M}_{A}, \overline{M_{B}}\right)=\delta_{A, B}
$$

for all $A, B \in \mathrm{Col}^{\Lambda}$. We refer to this pairing as the contravariant form. By [B1, Theorem 11] we have that

$$
\left(\widetilde{L}_{A}, T_{B}\right)=\delta_{A, B}
$$


for all $A, B \in \mathrm{Col}^{\Lambda}$. Note also by [B1, Lemma 3] that the form (.,.) has the property that

$$
(u x, y)=\left(x, u^{*} y\right)
$$

where $*: U \rightarrow U$ is the linear anti-automorphism with $E_{i}^{*}=F_{i}, F_{i}^{*}=E_{i}$ and $D_{i}^{*}=D_{i}^{-1}$.

The sesquilinear form $\langle.,$.$\rangle restricts to a form on V(\Lambda)$ which is characterized uniquely by sesquilinearity, the property (2.41) and the fact that $\left\langle v_{\Lambda}, v_{\Lambda}\right\rangle=1$. We call this the Shapovalov form on $V(\Lambda)$. Similarly the contravariant form $(.,$.$) induces a form on V(\Lambda)$, namely, the unique symmetric bilinear form $(.,$. on $V(\Lambda)$ such that (2.44) holds and $\left(v_{\Lambda}, v_{\Lambda}\right)=1$. In fact the Shapovalov form and the contravariant form on $V(\Lambda)$ are closely related:

Lemma 2.6. For vectors $v, w \in V(\Lambda)$ with $v$ of weight $\Lambda-\alpha$, we have that

(i) $\langle v, w\rangle=q^{\frac{1}{2}(2 \Lambda-\alpha, \alpha)}(\bar{v}, w)$;

(ii) $(v, w)=\overline{(\bar{w}, \bar{v})}$;

(iii) $\langle v, w\rangle=q^{(2 \Lambda-\alpha, \alpha)} \overline{\langle w, v\rangle}$.

Proof. The first two equalities follow by induction on $\operatorname{ht}(\alpha)$; for the induction step consider $v=F_{i} v^{\prime}$ for some $i \in I$ and $v^{\prime}$ of weight $\Lambda-\left(\alpha-\alpha_{i}\right)$ and use the defining properties (2.41) and (2.44). The third equality is a consequence of the first two.

The following result explains the relationship between the quasi-canonical and canonical bases of $F(\Lambda)$ and the usual canonical basis of the submodule $V(\Lambda)$ (which is Kashiwara's lower global crystal base). Recall the bijection from (2.33) which identifies the two natural choices of indexing set $\operatorname{Std}^{\Lambda}$ and $\operatorname{Rev}^{\Lambda}$ in this subject.

Theorem 2.7. The vectors $\left\{T_{A} \mid A \in \operatorname{Rev}^{\Lambda}\right\}$ give a basis for $V(\Lambda)$ which coincides with Lusztig's canonical basis. Moreover, for $A \in \operatorname{Std}_{\alpha}^{\Lambda}$, we have that

$$
P_{A}=q^{\frac{1}{2}(2 \Lambda-\alpha, \alpha)} T_{A^{\uparrow}} .
$$

Hence the vectors $\left\{P_{A} \mid A \in \operatorname{Std}^{\Lambda}\right\}$ also give a basis for $V(\Lambda)$ which coincides with Lusztig's canonical basis up to rescaling each vector by a suitable power of q. Finally,

for all $A, B \in \operatorname{Std}^{\Lambda}$.

$$
\left\langle P_{A}, D_{B}\right\rangle=\left(T_{A^{\uparrow}}, D_{B}\right)=\delta_{A, B}
$$

Proof. The first statement is a special case of [L4, Proposition 27.1.7]; see also [B1, Theorem 29] where the fact that

$$
\left(T_{A^{\uparrow}}, D_{B}\right)=\delta_{A, B}
$$

is derived at the same time.

Next we claim that the vectors $\left\{P_{A} \mid A \in \operatorname{Std}^{\Lambda}\right\}$ also give a basis for $V(\Lambda)$. Let $X$ denote the subspace of $F(\Lambda)$ spanned by the vectors $\left\{P_{A} \mid A \in \operatorname{Std}^{\Lambda}\right\}$. In view of Corollary 2.3 and (2.39), we have that

$$
X=\{v \in F(\Lambda) \mid\langle v, w\rangle=0 \text { for all } w \in \operatorname{ker} \pi\} .
$$


Combining this with (2.41) and the fact that $\operatorname{ker} \pi$ is a $U$-submodule of $F(\Lambda)$, it follows that $X$ is a $U$-submodule of $F(\Lambda)$ too. Since $X$ contains the vector $v_{\Lambda}$ (that is $P_{A^{\Lambda}}$ ) we deduce that $V(\Lambda) \subseteq X$. Finally the weight spaces of $V(\Lambda)$ and $X$ have the same dimensions, so we actually have that $V(\Lambda)=X$. This proves the claim.

For $A, B \in \operatorname{Std}^{\Lambda}$, we have by (2.39) that

$$
\left\langle P_{A}, D_{B}\right\rangle=\left\langle P_{A}, L_{B}\right\rangle=\delta_{A, B} .
$$

On the other hand, assuming in addition that $A$ is of weight $\Lambda-\alpha$, we have by Lemma 2.6(i) and (2.45) that

$$
\left\langle q^{\frac{1}{2}(2 \Lambda-\alpha, \alpha)} T_{A^{\uparrow}}, D_{B}\right\rangle=\left(T_{A^{\uparrow}}, D_{B}\right)=\delta_{A, B} .
$$

Comparing with (2.46) this shows that $P_{A}=q^{\frac{1}{2}(2 \Lambda-\alpha, \alpha)} T_{A^{\uparrow}}$.

Corollary 2.8. Suppose that $A \in \operatorname{Std}_{\alpha}^{\Lambda}$ and $B \in \mathrm{Col}_{\alpha}^{\Lambda}$ for some $\alpha \in Q_{+}$, and set $a:=\frac{1}{2}(2 \Lambda-\alpha, \alpha)$. Then, $d_{A, B}(q)=0$ unless $A \leq B \leq A^{\uparrow}$. Moreover, $d_{A, A}(q)=1, d_{A, A^{\uparrow}}(q)=q^{a}$, and $d_{A, B}(q)$ belongs to $q \mathbb{Z}[q] \cap q^{a-1} \mathbb{Z}\left[q^{-1}\right]$ for $A<B<A^{\uparrow}$.

Proof. We know by Theorem 2.7 that $P_{A}=q^{a} T_{A^{\uparrow}}$. By the definition of $T_{A^{\uparrow}}$ this shows that

$$
P_{A}=q^{a} M_{A^{\uparrow}}+\left(\mathrm{a} q^{a-1} \mathbb{Z}\left[q^{-1}\right] \text {-linear combination of } M_{B} \text { 's for } B<A^{\uparrow}\right) .
$$

Recalling (2.36), this shows that $d_{A, A^{\uparrow}}(q)=q^{a}, d_{A, B}(q)$ is zero unless $B \leq A^{\uparrow}$, and $d_{A, B}(q)$ belongs to $q^{a-1} \mathbb{Z}\left[q^{-1}\right]$ if $B<A^{\uparrow}$. On the other hand, we already know from (2.16) that $d_{A, A}(q)=1, d_{A, B}(q)$ is zero unless $B \geq A$, and $d_{A, B}(q)$ belongs to $q \mathbb{Z}[q]$ for $B>A$.

We mention finally that there is an analogue of Theorem 2.7 (and its corollary) in the twisted case. We only need to know one thing about this: if we define the quasi-canonical basis elements for $\widetilde{F}(\Lambda)$ by setting

$$
\widetilde{P}_{A}:=\sum_{B \in \mathrm{Col}^{\Lambda}} \widetilde{d}_{A, B}(q) \widetilde{M}_{B}
$$

where $\widetilde{d}_{A, B}(q)$ is the polynomial from (2.30), then $\left\{\widetilde{P}_{A} \mid A \in \operatorname{Rev}^{\Lambda}\right\}$ is again equal to Lusztig's canonical basis for $V(\Lambda)$ up to rescaling by suitable powers of $q$. In fact it happens that

$$
\widetilde{P}_{A}=P_{A \downarrow}
$$

for each $A \in \operatorname{Rev}^{\Lambda}$, which is the dual formula to the one in Theorem 2.5

2.7. Multipartitions. Almost every combinatorial definition so far has an alternative formulation using the language of $l$-multipartitions instead of columnstrict $\Lambda$-tableaux. This alternative language is particularly convenient in the case that $I$ is not bounded-below, indeed, it is the language used in almost all of the existing literature surrounding Ariki's categorification theorem. In this subsection, we want to explain the dictionary between the two languages.

To start with let $\mathscr{P}$ denote the set of all partitions $\lambda=\left(\lambda_{1}, \lambda_{2}, \ldots\right)$ in the usual sense, i.e. weakly decreasing sequences of non-negative integers. We write $|\lambda|$ for $\lambda_{1}+\lambda_{2}+\cdots$. We often identify $\lambda \in \mathscr{P}$ with its Young diagram drawn 
according to the usual English convention, i.e. rows are indexed $1,2,3, \ldots$ from top to bottom, columns are indexed $1,2,3, \ldots$ from left to right, and there is a box in the $i$ th row and $j$ th column whenever $j \leq \lambda_{i}$. Here for example is the Young diagram of $\lambda=(3,2)$ :

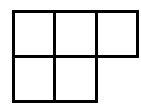

We stress that the Young diagram of a partition $\lambda$ plays a quite different role in this article to the diagram of the dominant weight $\Lambda \in P_{+}$from $\$ 2.1$.

By an $l$-multipartition we mean an $l$-tuple $\lambda=\left(\lambda^{(1)}, \ldots, \lambda^{(l)}\right) \in \mathscr{P} l$. The diagram of an $l$-multipartition $\lambda$ means the column vector containing the Young diagrams $\lambda^{(1)}, \ldots, \lambda^{(l)}$ in order from top to bottom. If the diagram of $\lambda$ contains $d$ boxes then we say that $\lambda$ is an $l$-multipartition of $d$. Here for example is the diagram of the 2-multipartition $((3,2,1),(3,1))$ of 10 with boxes filled by the integers $1, \ldots, 10$ :

$$
\left(\begin{array}{|l|l|l}
\hline 1 & 3 & 9 \\
\hline 2 & 4 & \\
\cline { 1 - 1 } 7 & \multicolumn{1}{|c}{} \\
\hline 5 & 6 & 10 \\
\hline 8 & \multicolumn{2}{|l}{} \\
\cline { 1 - 2 }
\end{array}\right.
$$

The addable and removable nodes of an $l$-multipartition $\lambda$ mean the places a box can be added to or removed from its diagram to again produce a valid diagram of an $l$-multipartition. Because of our convention of viewing the diagram of an l-multipartition as a column vector of Young diagrams, it makes sense to talk about one such node being above or below another in the diagram. In the above example, the removable node containing entry 9 is above the one containing entry 8.

Continue with $\Lambda$ fixed as in (2.1). Define the $\Lambda$-residue of box in the diagram of an $l$-multipartition to be the integer $m_{k}+j-i$, assuming the box is in the $i$ th row and $j$ th column of the $k$ th Young diagram. Let $\mathscr{P} \Lambda$ denote the set of all $l$-multipartitions $\lambda$ such that the $\Lambda$-residues of all the boxes in the diagram of $\lambda$ belong to $I$; we refer to elements of $\mathscr{P} \Lambda$ as $\Lambda$-multipartitions. There is then a bijection

$$
\mathrm{Col}^{\Lambda} \stackrel{\sim}{\rightarrow} \mathscr{P}^{\Lambda}, \quad A \mapsto \lambda(A)
$$

defined by letting $\lambda(A)=\left(\lambda^{(1)}, \ldots, \lambda^{(l)}\right)$ denote $l$-multipartition such that the parts of $\lambda^{(k)}$ are equal to the entries in the $k$ th column of the entry-wise difference $\left(A-A^{\Lambda}\right)$.

Using the bijection (2.50), it is usually routine to translate earlier definitions involving column-strict tableaux into equivalent notions involving multipartitions. To illustrate this, we explain how to lift the crystal operators from (2.25) to obtain maps

$$
\tilde{e}_{i}, \tilde{f}_{i}: \mathscr{P}^{\Lambda} \rightarrow \mathscr{P}^{\Lambda} \sqcup\{\odot\}
$$

Take $\lambda \in \mathscr{P}^{\Lambda}$ and $i \in I$. Enumerate the addable and removable nodes of $\Lambda$ residue $i$ in the diagram of $\lambda$ as $b_{1}, \ldots, b_{n}$ in order from top to bottom. Starting from the sequence $\left(\sigma_{1}, \ldots, \sigma_{n}\right)$ in which $\sigma_{r}$ is + if $b_{r}$ is addable or - if $b_{r}$ is removable, pass to the reduced $i$-signature by cancelling -+ pairs as in $\$ 2.4$. 
Then $\tilde{e}_{i}(\lambda)$ is obtained by removing a box from the position corresponding to the leftmost - in the reduced $i$-signature, or $\odot$ if no such - exists. Similarly $\tilde{f}_{i}(\lambda)$ is obtained by adding a box to the position corresponding to the rightmost + , or $\odot$ if no such + exists.

Under the bijection (2.50), the set $\operatorname{Std}^{\Lambda}$ of standard $\Lambda$-tableaux from $\$ 2.1$ corresponds to the set $\mathscr{R} \mathscr{P} \Lambda$ of restricted $\Lambda$-multipartitions, namely, the multipartitions $\lambda=\left(\lambda^{(1)}, \ldots, \lambda^{(l)}\right) \in \mathscr{P}^{\Lambda}$ such that

$$
\lambda_{j+m_{i}-m_{i+1}}^{(i)} \leq \lambda_{j}^{(i+1)}
$$

for each $i=1, \ldots, l-1$ and $j \geq 1$. This is the connected component of the crystal graph generated by the empty multipartition $\varnothing$. On the other hand the set $\operatorname{Rev}^{\Lambda}$ from $\oint 2.5$ corresponds to the set $\widetilde{\mathscr{R} P}^{\Lambda}$ of multipartitions $\lambda=\left(\lambda^{(1)}, \ldots, \lambda^{(l)}\right) \in \mathscr{P}^{\Lambda}$ such that

$$
\lambda_{j}^{(i)}+m_{i}-m_{i+1} \geq \lambda_{j}^{(i+1)}
$$

for each $i=1, \ldots, l-1$ and $j \geq 1$. The maps from (2.31) and (2.33) can be interpreted as mutually inverse bijections between the sets $\mathscr{R} \mathscr{P} \Lambda$ and $\widetilde{\mathscr{R} P}^{\Lambda}$.

2.8. Integral forms and specialization at $\boldsymbol{q}=\mathbf{1}$. In the rest of the article we work only at $q=1$. To explain how to specialize formally, let $\mathscr{A}:=\mathbb{Z}\left[q, q^{-1}\right]$ and $U_{\mathscr{A}}$ denote Lusztig's $\mathscr{A}$-form for $U$. This is the $\mathscr{A}$-subalgebra of $U$ generated by the quantum divided powers $E_{i}^{(r)}, F_{i}^{(r)}$, the elements $D_{i}, D_{i}^{-1}$, and the elements

$$
\left[\begin{array}{c}
D_{i} \\
r
\end{array}\right]:=\prod_{s=1}^{r} \frac{D_{i} q^{1-s}-D_{i}^{-1} q^{s-1}}{q^{s}-q^{-s}}
$$

for all $i$ and $r \geq 0$. The Hopf algebra structure on $U$ makes $U_{\mathscr{A}}$ into a Hopf algebra over $\mathscr{A}$. The bar involution and the map $\tau$ from (2.40) also restrict to well-defined maps on $U_{\mathscr{A}}$.

There are two natural $\mathscr{A}$-forms for $V(\Lambda)$, namely, the standard form $V(\Lambda)_{\mathscr{A}}$ which is obtained by applying $U_{\mathscr{A}}$ to the highest weight vector $v_{\Lambda}$, and the costandard form $V(\Lambda)_{\mathscr{A}}^{*}$ which is the dual lattice under the Shapovalov form:

$$
V(\Lambda)_{\mathscr{A}}^{*}:=\left\{v \in V(\Lambda) \mid\langle v, w\rangle \in \mathscr{A} \text { for all } w \in V(\Lambda)_{\mathscr{A}}\right\} \text {. }
$$

The costandard form $V(\Lambda)_{\mathscr{A}}^{*}$ is the $\mathscr{A}$-submodule of $V(\Lambda)$ spanned by either the dual-canonical basis or the standard monomial basis. Moreover, $V(\Lambda)_{\mathscr{A}}$, which is naturally a $U_{\mathscr{A}}$-submodule of $V(\Lambda)_{\mathscr{A}}^{*}$, is the $\mathscr{A}$-span of the quasicanonical basis $\left\{P_{A} \mid A \in \operatorname{Std}^{\Lambda}\right\}$. We also have an obvious $\mathscr{A}$-form $F(\Lambda)_{\mathscr{A}}$ for $F(\Lambda)$, namely, the $\mathscr{A}$-span of any of the four natural bases $\left\{M_{A}\right\},\left\{L_{A}\right\},\left\{T_{A}\right\}$ or $\left\{P_{A}\right\}$. The projection $\pi\left(F(\Lambda)_{\mathscr{A}}\right)$ is precisely the costandard form $V(\Lambda)_{\mathscr{A}}^{*}$ for $V(\Lambda)$. Similarly there is an $\mathscr{A}$-form $\widetilde{F}(\Lambda)_{\mathscr{A}}$ for $\widetilde{F}(\Lambda)$.

With these $\mathscr{A}$-forms in hand, we can finally specialize at $q=1$. Let $U_{\mathbb{Z}}$ be the Kostant $\mathbb{Z}$-form for the universal enveloping algebra of $\mathfrak{g l}_{I_{+}}(\mathbb{C})$, with 
Chevalley generators $e_{i}, f_{i}(i \in I)$. Let

$$
\begin{aligned}
F(\Lambda)_{\mathbb{Z}} & :=\mathbb{Z} \otimes_{\mathscr{A}} F(\Lambda)_{\mathscr{A}}, \\
\widetilde{F}(\Lambda)_{\mathbb{Z}} & :=\mathbb{Z} \otimes_{\mathscr{A}} \widetilde{F}(\Lambda)_{\mathscr{A}}, \\
V(\Lambda)_{\mathbb{Z}} & :=\mathbb{Z} \otimes_{\mathscr{A}} V(\Lambda)_{\mathscr{A}}, \\
V(\Lambda)_{\mathbb{Z}}^{*} & :=\mathbb{Z} \otimes_{\mathscr{A}} V(\Lambda)_{\mathscr{A}}^{*},
\end{aligned}
$$

viewing $\mathbb{Z}$ as an $\mathscr{A}$-module so that $q$ acts as 1 . These are naturally $U_{\mathbb{Z}}$-modules; the divided powers $e_{i}^{(r)}$ and $f_{i}^{(r)}$ act as $1 \otimes E_{i}^{(r)}$ and $1 \otimes F_{i}^{(r)}$, respectively. Of course $V(\Lambda)_{\mathbb{Z}}$ (resp. $V(\Lambda)_{\mathbb{Z}}^{*}$ ) is the standard (resp. costandard) form for the irreducible highest weight module for $\mathfrak{g l}_{I_{+}}(\mathbb{C})$ of highest weight $\Lambda$.

For the remainder of the article we will only be working at $q=1$, so it will not cause confusion to use the same notation $M_{A}, L_{A}, \ldots$ for the specializations of the basis elements $M_{A}, L_{A}, \ldots$ defined above at $q=1$. In particular, making this abuse of notation, $\left\{S_{A} \mid A \in \operatorname{Std}^{\Lambda}\right\}$ becomes precisely the classical standard monomial basis for $V(\Lambda)_{\mathbb{Z}}^{*}$, which can be constructed directly as above without going via quantum groups. The bases $\left\{P_{A} \mid A \in \operatorname{Std}^{\Lambda}\right\}$ and $\left\{D_{A} \mid A \in \operatorname{Std}^{\Lambda}\right\}$ are the usual canonical and dual-canonical bases of $V(\Lambda)_{\mathbb{Z}}$ and $V(\Lambda)_{\mathbb{Z}}^{*}$, respectively. Moreover these two bases are dual to each other under the Shapovalov form (which at $q=1$ coincides with the contravariant form).

\section{The CATEGORIFiCATION THEOREMS}

Now we categorify the spaces (2.54)-(2.57) using parabolic category $\mathcal{O}$ and degenerate cyclotomic Hecke algebras. Throughout the section we let $I$ be a non-empty set of consecutive integers, and fix all the other notation from section 2 according to this choice. Whenever we discuss parabolic category $\mathcal{O}$ or appeal to Schur-Weyl duality, we will assume further that $I$ is bounded-below.

3.1. Parabolic category $\mathcal{O}$. In this subsection, we assume that the index set $I$ is bounded-below. We want to recall a well known categorification of the

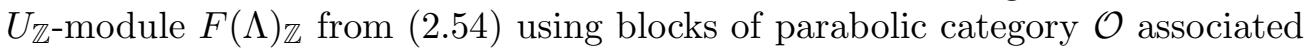
to the general linear Lie algebra. We refer the reader to [BK2, §4.4] for a more detailed treatment.

Since $I$ is bounded-below, there are only finitely many boxes in the diagram of $\Lambda$. Let $n$ denote this number. Also let $n_{i}:=m_{i}+1-\inf (I)$. This is the number of boxes in the $i$ th column of the diagram of $\Lambda$, so

$$
n=n_{1}+\cdots+n_{l} .
$$

We then have simply that

$$
F(\Lambda)_{\mathbb{Z}}=\bigwedge^{n_{1}} V_{\mathbb{Z}} \otimes \cdots \otimes \bigwedge^{n_{l}} V_{\mathbb{Z}}
$$

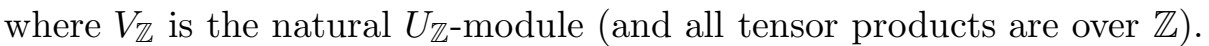

Consider the Lie algebra $\mathfrak{g}:=\mathfrak{g l}_{n}(\mathbb{C})$, with its standard Cartan subalgebra $\mathfrak{h}$ of diagonal matrices and its standard Borel subalgebra $\mathfrak{b}$ of upper triangular matrices. Let $\varepsilon_{1}, \ldots, \varepsilon_{n} \in \mathfrak{h}^{*}$ denote the standard coordinate functions, so $\varepsilon_{i}$ picks out the $i$ th diagonal entry of a diagonal matrix. Given any $\Lambda$-tableau $A$, we let $L(A)$ denote the usual irreducible highest weight module for $\mathfrak{g}$ of highest 
weight $a_{1} \varepsilon_{1}+\left(a_{2}+1\right) \varepsilon_{2}+\cdots+\left(a_{n}+n-1\right) \varepsilon_{n}$, where $a_{1}, \ldots, a_{n}$ is the columnreading of $A$, i.e. the sequence obtained by reading its entries down columns starting with the leftmost column. For $A, B \in \mathrm{Col}^{\Lambda}$, the irreducible modules $L(A), L(B)$ have the same central character if and only if $\operatorname{wt}(A)=\operatorname{wt}(B)$ according to (2.3).

For $\alpha \in Q_{+}$, let $\mathcal{O}_{\alpha}^{\Lambda}$ denote the category of all $\mathfrak{g}$-modules that are semisimple over $\mathfrak{h}$ and have a composition series with composition factors of the form $L(A)$ for $A \in \mathrm{Col}_{\alpha}^{\Lambda}$. When non-zero, $\mathcal{O}_{\alpha}^{\Lambda}$ is known by [B2, Theorem 2] to be a single block of the parabolic analogue of the Bernstein-Gelfand-Gelfand category $\mathcal{O}$ associated to the standard parabolic subalgebra $\mathfrak{p}$ of $\mathfrak{g}$ with Levi factor $\mathfrak{g l}_{n_{1}}(\mathbb{C}) \oplus \cdots \oplus \mathfrak{g l}_{n_{l}}(\mathbb{C})$. Hence

$$
\mathcal{O}^{\Lambda}:=\bigoplus_{\alpha \in Q_{+}} \mathcal{O}_{\alpha}^{\Lambda}
$$

is a sum of blocks of this parabolic category $\mathcal{O}$. The category $\mathcal{O}^{\Lambda}$ is a highest weight category with irreducible objects $\left\{L(A) \mid A \in \mathrm{Col}^{\Lambda}\right\}$, standard objects $\left\{M(A) \mid A \in \mathrm{Col}^{\Lambda}\right\}$ (which are parabolic Verma modules), projective indecomposable modules $\left\{P(A) \mid A \in \mathrm{Col}^{\Lambda}\right\}$ and indecomposable tilting modules $\left\{T(A) \mid A \in \mathrm{Col}^{\Lambda}\right\}$. The isomorphism classes of these four families of modules give four natural bases for the Grothendieck group $\left[\mathcal{O}^{\Lambda}\right]$ of the category $\mathcal{O}^{\Lambda}$.

We also have the usual duality $\circledast$ on $\mathcal{O}^{\Lambda}$ defined with respect to the antiautomorphism $*: \mathfrak{g} \rightarrow \mathfrak{g}$ mapping a matrix to its transpose. The irreducible modules are self-dual in the sense that

$$
L(A)^{\circledast} \cong L(A)
$$

for each $A \in \mathrm{Col}^{\Lambda}$.

As for any highest weight category, the projective indecomposable module $P(A)$ has a filtration whose sections are standard modules, and the multiplicity of the standard module $M(B)$ as a section of any such filtration, denoted $(P(A): M(B))$, is equal to the composition multiplicity $[M(B): L(A)]$, i.e.

$$
(P(A): M(B))=[M(B): L(A)] .
$$

This result is usually referred to as Bernstein-Gelfand-Gelfand reciprocity after BGG.

The module $T(A)$ is the unique (up to isomorphism) self-dual indecomposable module in $\mathcal{O}^{\Lambda}$ possessing a filtration by standard modules in which $M(A)$ appears at the bottom. There is a twisted version of BGG reciprocity for tilting modules which describes the multiplicities of standard modules in any standard filtration of $T(A)$. We refer to this as Arkhipov-Soergel reciprocity; see 4.3 below for the detailed references. To formulate the result, given $A \in \mathrm{Col}^{\Lambda}$, let $\widetilde{L}(A)$ denote the irreducible $\mathfrak{g}$-module of highest weight $a_{1} \varepsilon_{1}+\left(a_{2}+1\right) \varepsilon_{2}+\cdots+\left(a_{n}+n-1\right) \varepsilon_{n}$, where $a_{1}, \ldots, a_{n}$ is the reverse-columnreading of the entries of $A$, i.e. the sequence obtained by reading down columns starting with the rightmost column. Let

$$
\widetilde{\mathcal{O}}^{\Lambda}:=\bigoplus_{\alpha \in Q_{+}} \widetilde{\mathcal{O}}_{\alpha}^{\Lambda}
$$


where $\widetilde{\mathcal{O}}_{\alpha}^{\Lambda}$ is the category of all $\mathfrak{g}$-modules that are semisimple over $\mathfrak{h}$ and have a composition series with composition factors of the form $\widetilde{L}(A)$ for $A \in \mathrm{Col}_{\alpha}^{\Lambda}$. This is a single block of the parabolic category $\mathcal{O}$ associated to the standard parabolic subalgebra $\widetilde{\mathfrak{p}}$ with Levi factor $\mathfrak{g l}_{n_{l}}(\mathbb{C}) \oplus \cdots \oplus \mathfrak{g l}_{n_{1}}(\mathbb{C})$ (so $\widetilde{\mathfrak{p}}$ is conjugate to the opposite parabolic to $\mathfrak{p})$. In the highest weight category $\widetilde{\mathcal{O}}^{\Lambda}$, we have irreducible modules $\left\{\widetilde{L}(A) \mid A \in \mathrm{Col}^{\Lambda}\right\}$, standard modules $\left\{\widetilde{M}(A) \mid A \in \mathrm{Col}^{\Lambda}\right\}$ and projective indecomposable modules $\left\{\widetilde{P}(A) \mid A \in \mathrm{Col}^{\Lambda}\right\}$. Now ArkhipovSoergel reciprocity is the assertion that

$$
(T(A): M(B))=[\widetilde{M}(B): \widetilde{L}(A)]
$$

for all $A, B \in \mathrm{Col}^{\Lambda}$.

Returning to the discussion just of the category $\mathcal{O}^{\Lambda}$, for each $i \in I$ and $\alpha \in Q_{+}$there are some much-studied special projective functors (e.g. see BFK, BK1, [CR]

$$
\mathcal{O}_{\alpha}^{\Lambda} \underset{e_{i}}{\stackrel{f_{i}}{\rightleftarrows}} \mathcal{O}_{\alpha+\alpha_{i}}^{\Lambda}
$$

defined as follows: $f_{i}$ is defined on $\mathcal{O}_{\alpha}^{\Lambda}$ by tensoring with the natural $\mathfrak{g}$-module of column vectors then projecting onto $\mathcal{O}_{\alpha+\alpha_{i}}^{\Lambda} ; e_{i}$ is defined on $\mathcal{O}_{\alpha+\alpha_{i}}^{\Lambda}$ by tensoring with the dual of the natural module then projecting onto $\mathcal{O}_{\alpha}^{\Lambda}$. Taking the direct sum of these functors over all $\alpha \in Q_{+}$, we obtain endofunctors $e_{i}$ and $f_{i}$ of $\mathcal{O}^{\Lambda}$. These functors $e_{i}$ and $f_{i}$ are biadjoint. Hence they are both exact, so induce well-defined endomorphisms of the Grothendieck group $\left[\mathcal{O}^{\Lambda}\right]$. We note also that the functors $e_{i}$ and $f_{i}$ commute with the duality $\circledast$.

Now we can state the following foundational categorification theorem. This should be viewed as a translation of the Kazhdan-Lusztig conjecture for $\mathfrak{g}$ into the language of canonical bases, and is probably best described as "folk-lore" as it seems to have been independently (re-)discovered by many different people since the time of [L4].

Theorem 3.1. Identify the Grothendieck group $\left[\mathcal{O}^{\Lambda}\right]$ with the $U_{\mathbb{Z}}$-module $F(\Lambda)_{\mathbb{Z}}$ by identifying $[M(A)]$ with $M_{A}$ for each $A \in \mathrm{Col}^{\Lambda}$.

(i) The following equalities hold for all $A \in \mathrm{Col}^{\Lambda}$ :

(a) $[L(A)]=L_{A}$;

(b) $[P(A)]=P_{A}$;

(c) $[T(A)]=T_{A}$.

(ii) We have that $\operatorname{dim} \operatorname{Hom}_{\mathfrak{g}}(P, M)=\langle[P],[M]\rangle$ for each $P, M \in \mathcal{O}^{\Lambda}$ with $P$ projective.

(iii) The endomorphisms of the Grothendieck group induced by the exact functors $e_{i}$ and $f_{i}$ from (3.6) coincide with the action of the Chevalley generators $e_{i}$ and $f_{i}$ of $U_{\mathbb{Z}}$ for each $i \in I$.

(iv) For $A \in \mathrm{Col}^{\Lambda}$ and $i \in I$, the module $e_{i}\left(L(A)\right.$ ) (resp. $f_{i}(L(A))$ ) is nonzero if and only if $\tilde{e}_{i}(A) \neq \odot\left(\right.$ resp. $\left.\tilde{f}_{i}(A) \neq \odot\right)$, in which case it is a selfdual indecomposable module with irreducible socle and head isomorphic to $L\left(\tilde{e}_{i}(A)\right)$ (resp. $\left.L\left(\tilde{f}_{i}(A)\right)\right)$. 
Proof. The assertions (i)(a), (iii) and (iv) are verified in [BK2, Theorem 4.5]. Note (i)(a) implies that

$$
[M(B): L(A)]=d_{A, B}(1)
$$

where $d_{A, B}(1)$ is the polynomial from (2.16) evaluated at $q=1$. Using BGG reciprocity and (2.36) specialized at $q=1$ we therefore have that

$$
[P(A)]=\sum_{B \in \mathrm{Col}^{\Lambda}} d_{A, B}(1)[M(B)]=\sum_{B \in \mathrm{Col}^{\Lambda}} d_{A, B}(1) M_{B}=P_{A}
$$

giving (i)(b). There is also an analogue of (i)(a) in the twisted setup proved in [BK2, Theorem 4.5], so we have that

$$
[\widetilde{M}(B): \widetilde{L}(A)]=\widetilde{d}_{A, B}(1)
$$

where $\widetilde{d}_{A, B}(1)$ is the polynomial from (2.30) evaluated at $q=1$. Combining this with Arkhipov-Soergel reciprocity, [B1, Corollary 12] and the definition [B1, (5.8)], it follows that

$$
[T(A)]=\sum_{B \in \mathrm{Col}^{\Lambda}} \widetilde{d}_{A, B}(1)[M(B)]=\sum_{B \in \mathrm{Col}^{\Lambda}} \widetilde{d}_{A, B}(1) M_{B}=T_{A},
$$

giving (i)(c). Finally, (ii) is clear from (i)(a), (i)(b), (2.39) specialized at $q=1$, and the observation that $\operatorname{dim} \operatorname{Hom}_{\mathfrak{g}}(P(A), L(B))=\delta_{A, B}$.

In the rest of the section, we are going to transfer this categorification theorem to degenerate cyclotomic Hecke algebras by applying Schur-Weyl duality for higher levels. We record two other useful lemmas about parabolic category $\mathcal{O}$, the first of which originates in work of Irving. It explains the representation theoretic significance of the subset $\operatorname{Std}^{\Lambda}$ of $\mathrm{Col}^{\Lambda}$.

Lemma 3.2. For $A \in \mathrm{Col}^{\Lambda}$ the following are equivalent:

(i) the projective indecomposable module $P(A)$ is injective in $\mathcal{O}^{\Lambda}$;

(ii) $P(A)$ is self-dual, i.e. $P(A) \circledast{ }^{\circledast} \cong P(A)$;

(iii) $L(A)$ embeds into $M(B)$ for some $B \in \mathrm{Col}^{\Lambda}$;

(iv) $A \in \operatorname{Std}^{\Lambda}$.

Proof. See [BK3, Theorem 4.8].

Lemma 3.3. For $A \in \operatorname{Std}^{\Lambda}$ the projective indecomposable module $P(A)$ is isomorphic to the tilting module $T\left(A^{\uparrow}\right)$. Moreover the standard module $M\left(A^{\uparrow}\right)$ has irreducible socle isomorphic to $L(A)$.

Proof. By Theorem $3.1(\mathrm{i})$ and (2.36), the multiplicity $(P(A): M(B))$ is equal to $d_{A, B}(1)$. Hence if $A \in \operatorname{Std}^{\Lambda}$ we deduce from Corollary 2.8 that $M\left(A^{\uparrow}\right)$ appears as a section of a standard filtration of $P(A)$ with multiplicity one, and all other $M(B)$ 's arising satisfy $B<A^{\uparrow}$. Since standard filtrations can always be ordered so that the most dominant section appears at the bottom, this means that $M\left(A^{\uparrow}\right)$ embeds into $P(A)$. As $P(A)$ is self-dual by Lemma 3.2, we deduce that $P(A) \cong T\left(A^{\uparrow}\right)$ by the definition of the latter module. Moreover $P(A)$ has irreducible head $L(A)$, hence by self-duality it also has irreducible socle isomorphic to $L(A)$. Hence $M\left(A^{\uparrow}\right)$ must also have irreducible socle isomorphic to $L(A)$. 
3.2. Degenerate cyclotomic Hecke algebras and blocks. In this subsection, we allow $I$ to be arbitrary; from the Hecke algebra point of view the most important case is when $I=\mathbb{Z}$.

For $d \geq 0$, let $H_{d}$ be the degenerate affine Hecke algebra from [D]. As a vector space,

$$
H_{d}=\mathbb{C}\left[x_{1}, \ldots, x_{d}\right] \otimes \mathbb{C} S_{d},
$$

the tensor product of a polynomial ring in variables $x_{1}, \ldots, x_{d}$ with the group algebra of the symmetric group. Multiplication is defined so that $\mathbb{C}\left[x_{1}, \ldots, x_{d}\right]=$ $\mathbb{C}\left[x_{1}, \ldots, x_{d}\right] \otimes 1$ and $\mathbb{C} S_{d}=1 \otimes \mathbb{C} S_{d}$ are subalgebras, and in addition

$$
s_{r} x_{s}=x_{s} s_{r} \text { if } s \neq r, r+1, \quad s_{r} x_{r+1}=x_{r} s_{r}+1, \quad x_{r+1} s_{r}=s_{r} x_{r}+1 .
$$

Here $s_{r}$ denotes the $r$ th basic transposition.

Suppose we are given $l \geq 0$ and an $l$-tuple $\boldsymbol{m}=\left(m_{1}, \cdots, m_{l}\right)$ of integers. Introduce the corresponding degenerate cyclotomic Hecke algebra of level $l$ :

$$
H_{d}^{m}:=H_{d} /\left\langle\left(x_{1}-m_{1}\right) \cdots\left(x_{1}-m_{l}\right)\right\rangle .
$$

A basic result is that the vectors

$$
\left\{x_{1}^{r_{1}} \cdots x_{d}^{r_{d}} w \mid 0 \leq r_{1}, \ldots, r_{d}<l, w \in S_{d}\right\}
$$

give a basis for $H_{d}^{\boldsymbol{m}}$, hence $\operatorname{dim} H_{d}^{\boldsymbol{m}}=l^{d} d$ !; see e.g. [BK3, Lemma 3.5] or [K, Theorem 7.5.6] for two possible proofs. Any finite dimensional $H_{d}^{m}$-module $M$ decomposes into weight spaces

$$
M=\bigoplus_{i \in \mathbb{Z}^{d}} M_{i}
$$

where $M_{\boldsymbol{i}}:=\left\{v \in M \mid\left(x_{r}-i_{r}\right)^{N} v=0\right.$ for all $r=1, \ldots, d$ and $\left.N \gg 0\right\}$. We have appealed here to a basic fact, namely, that the eigenvalues of the $x_{r}$ 's are necessarily integers; see e.g. [K, Lemma 7.1.2] for a proof. The formal character ch $M$ of $M$ can then be defined as

$$
\operatorname{ch} M:=\sum_{\boldsymbol{i} \in \mathbb{Z}^{d}} \operatorname{dim} M_{\boldsymbol{i}} \cdot \boldsymbol{i},
$$

which is an element of the free $\mathbb{Z}$-module with basis $\left\{\boldsymbol{i} \mid \boldsymbol{i} \in \mathbb{Z}^{d}\right\}$. Two finite dimensional $H_{d}^{m}$-modules are equal in the Grothendieck group if and only if their formal characters are equal; see $\mathrm{K}$, Theorem 5.3.1].

Considering the decomposition (3.9) for the regular module, it follows that there is a system

$$
\left\{e(\boldsymbol{i}) \mid \boldsymbol{i} \in \mathbb{Z}^{d}\right\}
$$

of mutually orthogonal idempotents in $H_{d}^{\boldsymbol{m}}$ such that $e(\boldsymbol{i}) M=M_{\boldsymbol{i}}$ for each finite dimensional module $M$. In fact, each $e(\boldsymbol{i})$ lies in the commutative subalgebra generated by $x_{1}, \ldots, x_{d}$, all but finitely many of the $e(\boldsymbol{i})$ 's are necessarily zero, and their sum is the identity element in $H_{d}^{m}$. By [B2, Theorem 1], the center $Z\left(H_{d}^{\boldsymbol{m}}\right)$ consists of all symmetric polynomials in $x_{1}, \ldots, x_{d}$. Hence, the sum of the $e(\boldsymbol{i})$ 's for $\boldsymbol{i}$ running over any $S_{d^{-}}$-orbit in $\mathbb{Z}^{d}$ is either zero or it is a primitive central idempotent in $H_{d}^{m}$. 
Now we pick a dominant weight $\Lambda \in P_{+}$of level $l$ and take $m_{1}, \ldots, m_{l}$ to be the integers defined by the decomposition (2.1). Given $\alpha \in Q_{+}$, let $d:=\operatorname{ht}(\alpha)$ and let

$$
e_{\alpha}:=\sum_{\boldsymbol{i} \in I^{\alpha}} e(\boldsymbol{i}) \in H_{d}^{\boldsymbol{m}}
$$

As remarked at the end of the previous paragraph, the non-zero $e_{\alpha}$ 's are the primitive central idempotents in $H_{d}^{m}$. Define

$$
H_{\alpha}^{\Lambda}:=e_{\alpha} H_{d}^{m} \text {. }
$$

The algebra $H_{\alpha}^{\Lambda}$ is either zero or it is a single block of $H_{d}^{m}$, and $\left\{e(\boldsymbol{i}) \mid \boldsymbol{i} \in I^{\alpha}\right\}$ is a system of mutually orthogonal idempotents summing to the identity in $H_{\alpha}^{\Lambda}$. Finally introduce the algebra

$$
H^{\Lambda}:=\bigoplus_{\alpha \in Q_{+}} H_{\alpha}^{\Lambda}
$$

For infinite $I$, this is a locally unital but not unital algebra, as it is a direct sum of infinitely many non-zero finite dimensional algebras. If $I=\mathbb{Z}$ then we have simply that

$$
H^{\Lambda}=\bigoplus_{d \geq 0} H_{d}^{m}
$$

However if $I \neq \mathbb{Z}$ then equality does not hold here: there always exist blocks of $H_{d}^{m}$ for sufficiently large $d$ that cannot be realized as $H_{\alpha}^{\Lambda}$ 's for any $\alpha \in Q_{+}$ (we recall $Q_{+}$depends implicitly on the index set $I$ ).

3.3. Grothendieck groups, induction and restriction. Denote the category of finite dimensional (resp. finitely generated projective) left $H_{\alpha}^{\Lambda}$-modules by $\operatorname{Rep}\left(H_{\alpha}^{\Lambda}\right)$ (resp. $\left.\operatorname{Proj}\left(H_{\alpha}^{\Lambda}\right)\right)$. The Grothendieck group $\left[\operatorname{Rep}\left(H_{\alpha}^{\Lambda}\right)\right]$ (resp. $\left.\left[\operatorname{Proj}\left(H_{\alpha}^{\Lambda}\right)\right]\right)$ is the free abelian group with basis given by the isomorphism classes of irreducible modules (resp. projective indecomposable modules). Sometimes it is convenient to work with all blocks at once: let $\operatorname{Rep}\left(H^{\Lambda}\right)\left(\operatorname{resp} \cdot \operatorname{Proj}\left(H^{\Lambda}\right)\right)$ denote category of all finite dimensional (resp. finitely generated projective) locally unital left modules over the locally unital algebra $H^{\Lambda}$ from (3.14). The Grothendieck groups of these categories then decompose into blocks as

$$
\begin{aligned}
{\left[\operatorname{Rep}\left(H^{\Lambda}\right)\right] } & =\bigoplus_{\alpha \in Q_{+}}\left[\operatorname{Rep}\left(H_{\alpha}^{\Lambda}\right)\right], \\
{\left[\operatorname{Proj}\left(H^{\Lambda}\right)\right] } & =\bigoplus_{\alpha \in Q_{+}}\left[\operatorname{Proj}\left(H_{\alpha}^{\Lambda}\right)\right] .
\end{aligned}
$$

We have the Cartan pairing

$$
\langle., .\rangle:\left[\operatorname{Proj}\left(H^{\Lambda}\right)\right] \times\left[\operatorname{Rep}\left(H^{\Lambda}\right)\right] \rightarrow \mathbb{Z}
$$

defined by setting $\langle[P],[M]\rangle:=\operatorname{dim}_{H^{\Lambda}}(P, M)$ for $P \in \operatorname{Proj}\left(H^{\Lambda}\right)$ and $M \in$ $\operatorname{Rep}\left(H^{\Lambda}\right)$. Of course different blocks are orthogonal under this pairing.

There is an obvious embedding $H_{d} \hookrightarrow H_{d+1}$ which in view of (3.8) factors through the quotients to induce an embedding $\iota: H_{d}^{m} \hookrightarrow H_{d+1}^{m}$. This map has the property that

$$
\iota\left(e\left(i_{1}, \ldots, i_{d}\right)\right)=\sum_{i \in \mathbb{Z}} e\left(i_{1}, \ldots, i_{d}, i\right)
$$


for any $i_{1}, \ldots, i_{d} \in \mathbb{Z}$. Given $i \in I$ and $\alpha \in Q_{+}$of height $d$, we can compose $\iota$ on one side with the embedding $H_{\alpha}^{\Lambda} \hookrightarrow H_{d}^{m}$ and on the other side with the projection $H_{d+1}^{m} \rightarrow H_{\alpha+\alpha_{i}}^{\Lambda}$ defined by multiplication by the block idempotent $e_{\alpha+\alpha_{i}}$, to obtain a non-unital algebra homomorphism

$$
\iota_{\alpha, \alpha_{i}}: H_{\alpha}^{\Lambda} \rightarrow H_{\alpha+\alpha_{i}}^{\Lambda} .
$$

By (3.16), the map $\iota_{\alpha, \alpha_{i}}$ maps the identity element $e_{\alpha}$ of $H_{\alpha}^{\Lambda}$ to the idempotent

$$
e_{\alpha, \alpha_{i}}:=\sum_{\boldsymbol{i} \in I^{\alpha+\alpha_{i}} i_{d+1}=i} e(\boldsymbol{i}) \in H_{\alpha+\alpha_{i}}^{\Lambda} .
$$

To the homomorphism $\iota_{\alpha, \alpha_{i}}$, we associate the induction and restriction functors

$$
\operatorname{Rep}\left(H_{\alpha}^{\Lambda}\right) \underset{e_{i}}{\stackrel{f_{i}}{\rightleftarrows}} \operatorname{Rep}\left(H_{\alpha+\alpha_{i}}^{\Lambda}\right)
$$

So on an object $M \in \operatorname{Rep}\left(H_{\alpha+\alpha_{i}}^{\Lambda}\right)$ we define $e_{i} M$ to be the the vector space $e_{\alpha, \alpha_{i}} M$ viewed as an $H_{\alpha}^{\Lambda}$-module via the homomorphism $\iota_{\alpha, \alpha_{i}}$, and on an object $M \in \operatorname{Rep}\left(H_{\alpha}^{\Lambda}\right)$ we define $f_{i} M$ to be $H_{\alpha+\alpha_{i}}^{\Lambda} e_{\alpha, \alpha_{i}} \otimes_{H_{\alpha}^{\Lambda}} M$. Taking the direct sum of these functors over all $\alpha \in Q_{+}$, we obtain endofunctors $e_{i}$ and $f_{i}$ of $\operatorname{Rep}\left(H^{\Lambda}\right)$. It is known that these functors $e_{i}$ and $f_{i}$ are biadjoint; see [K, Lemma 8.2.2]. Hence they induce endomorphisms of the Grothendieck groups $\left[\operatorname{Rep}\left(H^{\Lambda}\right)\right]$ and $\left[\operatorname{Proj}\left(H^{\Lambda}\right)\right]$, and these induced endomorphisms are biadjoint with respect to the Cartan pairing.

We note finally that $H_{d}^{m}$ possesses an anti-automorphism $*$ with $s_{r}^{*}=s_{r}$ and $x_{r}^{*}=x_{r}$ for each $r$. This anti-automorphism leaves each $H_{\alpha}^{\Lambda}$ invariant so gives an anti-automorphism

$$
*: H_{\alpha}^{\Lambda} \rightarrow H_{\alpha}^{\Lambda}
$$

for each $\alpha \in Q_{+}$. Using this we can introduce a natural duality $\circledast$ on finite dimensional left $H_{\alpha}^{\Lambda}$-modules: $M^{\circledast}$ denotes the dual space with left action defined via the anti-automorphism $*$. This duality fixes the irreducible modules. The functors $e_{i}$ and $f_{i}$ commute with $\circledast$; see $[\mathrm{K}$, Lemma 8.2.2] again.

3.4. Specht modules. We want to define one important family of $H_{\alpha}^{\Lambda}$-modules, namely, the Specht modules

$$
\left\{S(A) \mid A \in \operatorname{Col}_{\alpha}^{\Lambda}\right\}
$$

following [BK3, (6.20)]; this approach to defining Specht modules by induction from level one goes back at least to Vazirani. Given $d=d^{\prime}+d^{\prime \prime}$, we have the obvious natural embedding of $H_{d^{\prime}, d^{\prime \prime}}:=H_{d^{\prime}} \otimes H_{d^{\prime \prime}}$ into $H_{d}$. If $M^{\prime}$ is an $H_{d^{\prime}}$-module and $M^{\prime \prime}$ is an $H_{d^{\prime \prime}}$-module, we define

$$
M^{\prime} \circ M^{\prime \prime}:=H_{d} \otimes_{H_{d^{\prime}, d^{\prime \prime}}}\left(M^{\prime} \otimes M^{\prime \prime}\right),
$$

where $M^{\prime} \otimes M^{\prime \prime}$ denotes the outer tensor product.

For any partition $\lambda$ of $d$ let $S(\lambda)$ denote the corresponding irreducible $\mathbb{C} S_{d^{-}}$ module. For any $m \in \mathbb{C}$ there is an evaluation homomorphism

$$
\mathrm{ev}_{m}: H_{d} \rightarrow \mathbb{C} S_{d}
$$


mapping each $s_{r}$ to $s_{r}$ and $x_{1}$ to $m$. Let $\mathrm{ev}_{m}^{*}(S(\lambda))$ denote the lift of $S(\lambda)$ to an $H_{d}$-module via this homomorphism ev ${ }_{m}$. Now suppose that $A \in \operatorname{Col}_{\alpha}^{\Lambda}$ and let $\lambda(A)=\left(\lambda^{(1)}, \ldots, \lambda^{(l)}\right)$ be the corresponding $\Lambda$-multipartition according to the bijection (2.50). Then we define

$$
S(A):=\mathrm{ev}_{m_{1}}^{*}\left(S\left(\lambda^{(1)}\right)\right) \circ \cdots \circ \mathrm{ev}_{m_{l}}^{*}\left(S\left(\lambda^{(l)}\right)\right) .
$$

As written, this is a finite dimensional left $H_{d}$-module, but it is not hard to check that $\left(x_{1}-m_{1}\right) \cdots\left(x_{1}-m_{l}\right)$ acts as zero, hence $S(A)$ can naturally be viewed as an $H_{d}^{m}$-module. A straightforward character calculation (see e.g. [B2, p.247]) shows moreover that $S(A)$ belongs to the block $e_{\alpha}$, i.e. $e_{\alpha} S(A)=S(A)$. Hence $S(A)$ is a unital $H_{\alpha}^{\Lambda}$-module.

Remark 3.4. The Specht module $S(A)$ just defined is the same as the cell module $S(\lambda)$ that is defined in [AMR, $\S 6]$ for the multipartition $\lambda:=\lambda(A)$. This is the degenerate analogue of the Specht module of [DJM, (3.28)]. One could also choose to work everywhere in terms of the modules

$$
\widetilde{S}(A):=\mathrm{ev}_{m_{l}}^{*}\left(S\left(\lambda^{(l)}\right)\right) \circ \cdots \circ \mathrm{ev}_{m_{1}}^{*}\left(S\left(\lambda^{(1)}\right)\right),
$$

using the same notation as (3.24). Note by [K, Corollary 3.7.5] that

$$
\widetilde{S}(A) \cong S(A)^{\circledast} \text {. }
$$

In particular, this means that $S(A)$ and $\widetilde{S}(A)$ have the same formal characters, so are equal in the Grothendieck group.

3.5. Schur-Weyl duality for level $\boldsymbol{l}$. In this subsection we assume once more that $I$ is bounded-below. We are going to recall the Schur-Weyl duality from [BK3] which links the representation theory of the algebra $H^{\Lambda}$ from (3.14) to the parabolic category $\mathcal{O}^{\Lambda}$ from (3.1). Let $T_{d}$ denote the $d$ th tensor power of the natural $\mathfrak{g}$-module of column vectors. For any $\mathfrak{g}$-module $M$, there is a natural right action of the degenerate affine Hecke algebra $H_{d}$ on $M \otimes T_{d}$ commuting with the left action of $\mathfrak{g}$. This action is defined by letting elements of $S_{d}$ act by permuting tensors in $T_{d}$ like in classical Schur-Weyl duality. The remaining generator $x_{1}$ acts as $\Omega \otimes 1^{\otimes(d-1)}$, where $\Omega:=\sum_{i, j=1}^{n} e_{i, j} \otimes e_{j, i} \in \mathfrak{g} \otimes \mathfrak{g}$ (the "trace form").

Now recall the ground-state tableau $A^{\Lambda}$ from 92.1 . It is the only columnstrict $\Lambda$-tableau of weight $\Lambda$, hence $P\left(A^{\Lambda}\right) \cong M\left(A^{\Lambda}\right) \cong L\left(A^{\Lambda}\right)$. This module plays a very special role: according to Theorem 3.1 its isomorphism class in the Grothendieck group $\left[\mathcal{O}^{\Lambda}\right]$ coincides with the highest weight vector $v_{\Lambda} \in F(\Lambda)_{\mathbb{Z}}$. For $\alpha \in Q_{+}$with $\operatorname{ht}(\alpha)=d$, we define $T_{\alpha}^{\Lambda}$ to be the projection of the tensor product $P\left(A^{\Lambda}\right) \otimes T_{d}$ onto the block $\mathcal{O}_{\alpha}^{\Lambda}$. Equivalently, recalling the functor $f_{i}$ from (3.6), we have that

$$
T_{\alpha}^{\Lambda}=\bigoplus_{i \in I^{\alpha}} f_{i_{d}} \cdots f_{i_{1}}\left(P\left(A^{\Lambda}\right)\right) .
$$

Then set

$$
T^{\Lambda}:=\bigoplus_{\alpha \in Q_{+}} T_{\alpha}^{\Lambda} .
$$

By a prinjective module we mean a module that is both projective and injective. By Lemma 3.2, we know that the modules $\left\{P(A) \mid A \in \operatorname{Std}^{\Lambda}\right\}$ give a full set 
of representatives for the isomorphism classes of prinjective indecomposable modules in the category $\mathcal{O}^{\Lambda}$.

Lemma 3.5. The module $T^{\Lambda}$ is a prinjective generator for $\mathcal{O}^{\Lambda}$, i.e. it is a direct sum of copies of the prinjective indecomposable modules $\left\{P(A) \mid A \in \operatorname{Std}^{\Lambda}\right\}$, and each of these modules appears as a summand of $T^{\Lambda}$ with multiplicity at least one.

Proof. We note that $P\left(A^{\Lambda}\right)$ is prinjective, and tensoring with finite dimensional modules preserves projective and injective modules. Hence each $P\left(A^{\Lambda}\right) \otimes T_{d}$ is prinjective, so all their summands are too. This shows that all indecomposable summands of $T^{\Lambda}$ are of the form $P(A)$ for some $A \in \operatorname{Std}^{\Lambda}$. The fact that each of these appears in $T^{\Lambda}$ with multiplicity at least one is established in BK3, Corollary 4.6].

Now we discuss the endomorphism algebra of $T_{\alpha}^{\Lambda}$ for $\alpha \in Q_{+}$. Letting $d:=\operatorname{ht}(\alpha)$, BK3, Corollary 5.12] shows that the natural right action of $H_{d}$ on $P\left(A^{\Lambda}\right) \otimes T_{d}$ defined above factors through the quotient to make $P\left(A^{\Lambda}\right) \otimes T_{d}$ into a well-defined right $H_{d}^{m}$-module. Moreover by [B2, Lemma 5.8] the canonical g-equivariant projection of $P\left(A^{\Lambda}\right) \otimes T_{d}$ onto the summand $T_{\alpha}^{\Lambda}$ coincides with the action of the central idempotent $e_{\alpha} \in H_{d}^{m}$. Hence $T_{\alpha}^{\Lambda}$ is naturally a $\left(U(\mathfrak{g}), H_{\alpha}^{\Lambda}\right)$-bimodule. The following theorem should be viewed as a slight reformulation of the main result of Schur-Weyl duality for level $l$ from [BK3]; it was formulated on a block-by-block basis like this already in B2, Theorem $5.9]$.

Theorem 3.6. The natural right action of $H_{\alpha}^{\Lambda}$ on $T_{\alpha}^{\Lambda}$ induces an algebra isomorphism between $H_{\alpha}^{\Lambda}$ and $\operatorname{End}_{\mathfrak{g}}\left(T_{\alpha}^{\Lambda}\right)^{\mathrm{op}}$.

Proof. This is a consequence of [BK3, Theorem 5.13], [BK3, Corollary 6.7] and the description of the idempotent $e_{\alpha}$ from [B2, Lemma 5.8] mentioned just before the statement of the theorem.

Recalling (3.28) and (3.14), we have that $T^{\Lambda}$ is a $\left(U(\mathfrak{g}), H^{\Lambda}\right)$-bimodule, and Theorem 3.6 allows us to identify

$$
H^{\Lambda}=\operatorname{End}_{\mathfrak{g}}\left(T^{\Lambda}\right)^{\mathrm{op}} .
$$

Now introduce the Schur functors

$$
\begin{array}{r}
\pi:=\operatorname{Hom}_{\mathfrak{g}}\left(T^{\Lambda}, ?\right): \mathcal{O}^{\Lambda} \rightarrow \operatorname{Rep}\left(H^{\Lambda}\right), \\
\pi^{*}:=T^{\Lambda} \otimes_{H^{\Lambda}} ?: \operatorname{Rep}\left(H^{\Lambda}\right) \rightarrow \mathcal{O}^{\Lambda} .
\end{array}
$$

Because of (3.29), the functor $\pi$ is a quotient functor in the general sense of $\mathrm{G}$, $\S$ III.1], so we are in a well understood situation. In particular, the functor $\pi$ is exact and $\pi^{*}$ is left adjoint to $\pi$, hence $\pi^{*}$ sends projectives to projectives. The following theorem collates all the other important known facts about these functors that we will need later on.

Theorem 3.7. For $A \in \mathrm{Col}^{\Lambda}, \pi(L(A))$ is non-zero if and only if $A \in \operatorname{Std}^{\Lambda}$. Moreover:

(i) The modules $\left\{\pi(L(A)) \mid A \in \operatorname{Std}^{\Lambda}\right\}$ give a complete set of pairwise inequivalent irreducible $H^{\Lambda}$-modules. 
(ii) For $A \in \operatorname{Std}^{\Lambda}$, the module $\pi(P(A))$ is the projective cover of $\pi(L(A))$, and $\pi^{*}(\pi(P(A))) \cong P(A)$.

(iii) For $A \in \mathrm{Col}^{\Lambda}$, we have that $\pi(M(A)) \cong S(A)$.

(iv) There is an isomorphism of functors $f_{i} \circ \pi \cong \pi \circ f_{i}$, where $f_{i}$ on the left hand side comes from (3.19) and $f_{i}$ on the right hand side comes from (3.6).

(v) For any $M, N \in \mathcal{O}^{\Lambda}$ such that all composition factors of the head of $M$ and the socle of $N$ are of the form $L(A)$ for $A \in \operatorname{Std}^{\Lambda}$, the functor $\pi$ defines a vector space isomorphism

$$
\operatorname{Hom}_{\mathfrak{g}}(M, N) \stackrel{\sim}{\rightarrow} \operatorname{Hom}_{H^{\Lambda}}(\pi(M), \pi(N)) .
$$

(vi) The functor $\pi$ is fully faithful both on projectives and on tilting modules.

Proof. The first statement is clear from Lemma 3.5. Given that, (i) and (v) are general facts about quotient functors; see [BDK, Corollary 3.1e] and [BDK, Corollary 3.1c], respectively. Also (iii) is [BK3, Theorem 6.12], (iv) follows from [BK3, Lemma 5.16].

For (ii), let $e \in \operatorname{End}_{\mathfrak{g}}\left(T^{\Lambda}\right)^{\text {op }}$ be an idempotent such that $\left(T^{\Lambda}\right) e \cong P(A)$; such idempotents exist by Lemma 3.5. By (3.29) and Fitting's lemma, $e$ is a primitive idempotent in $H^{\Lambda}$, hence $\left(H^{\Lambda}\right) e$ is a projective indecomposable module. Clearly this is isomorphic to $\pi(P(A))$, which maps surjectively onto $\pi(L(A))$. Hence $\pi(P(A))$ is the projective cover of $\pi(L(A))$. It remains to show that

$$
\pi^{*}(\pi(P(A))) \cong P(A)
$$

Since $P(A)$ has a standard filtration, Lemma 3.2 implies that all constituents of the socle of $P(A)$ are of the form $L(B)$ for $B \in \operatorname{Std}^{\Lambda}$. Using this, BDK, Theorem 3.1d] and [BDK, Lemma 3.1f] we get that $\pi^{*}(\pi(P(A))) \cong \pi^{*}\left(\left(H^{\Lambda}\right) e\right) \cong$ $\left(T^{\Lambda}\right) e \cong P(A)$.

Finally, for (vi), the fact that $\pi$ is fully faithful on projectives is BK3, Theorem 6.10]. The fact that it is fully faithful on tilting modules is a consequence of $(\mathrm{v})$, since all composition factors of the socle and head of any tilting module are of the form $L(A)$ for $A \in \operatorname{Std}^{\Lambda}$. For the latter statement, tilting modules are self-dual, so it suffices to verify it for the socle. Now use the fact that tilting modules have a filtration by standard modules, and all composition factors of the socle of any standard module are of the form $L(A)$ for $A \in \operatorname{Std}^{\Lambda}$ according to Lemma 3.2 (iii).

3.6. The main categorification theorem. Now we use Schur-Weyl duality to deduce the degenerate analogue of Ariki's categorification theorem from Theorem 3.1, working now with an arbitrary index set $I$. The first job is to recover the classification of the irreducible $H^{\Lambda}$-modules. The same strategy was noted already in [BK3, Theorem 6.15].

Theorem 3.8. For $A \in \operatorname{Std}^{\Lambda}$, the Specht module $S(A)$ has irreducible head denoted $D(A)$. The modules $\left\{D(A) \mid A \in \operatorname{Std}^{\Lambda}\right\}$ give a full set of pairwise inequivalent irreducible $H^{\Lambda}$-modules.

Proof. Suppose first that $I$ is bounded-below and let $\pi$ be the Schur functor from (3.30). By Theorem 3.7(i) the modules $\left\{\pi(L(A)) \mid A \in \operatorname{Std}^{\Lambda}\right\}$ give a 
complete set of irreducible modules. Moreover by Theorem 3.7(iii) we know that $\pi(M(A)) \cong S(A)$. Therefore we just need to show that $\pi(M(A))$ has irreducible head isomorphic to $\pi(L(A))$. This follows by Theorem 3.7(v) and the fact that $M(A)$ has irreducible head isomorphic to $L(A)$ : for any $B \in \operatorname{Std}^{\Lambda}$

$$
\operatorname{Hom}_{H^{\Lambda}}(\pi(M(A)), \pi(L(B))) \cong \operatorname{Hom}_{\mathfrak{g}}(M(A), L(B))
$$

which is one dimensional if $B=A$ and zero otherwise.

Now assume that $I$ is not bounded-below. It is enough to show for each $\alpha \in Q_{+}$that $S(A)$ has irreducible head denoted $D(A)$ for each $A \in \operatorname{Std}_{\alpha}^{\Lambda}$, and that the irreducible modules $\left\{D(A) \mid A \in \operatorname{Std}_{\alpha}^{\Lambda}\right\}$ give a complete set of irreducible $H_{\alpha}^{\Lambda}$-modules. Given $\alpha$, we pick $k$ such that $\alpha$ is a sum of simple roots of the form $\alpha_{i}$ for $i \geq k$. Then the block $H_{\alpha}^{\Lambda}$ already appears as a block of the algebra $H^{\Lambda}$ defined with respect to the bounded-below index set $I_{\geq k}$. Moreover for $A \in \operatorname{Std}_{\alpha}^{\Lambda}$, it is clear from the definition that the Specht module $S(A)$ is the same module regardless of whether we are considering $I$ or $I_{\geq k}$. Hence we are done by the previous paragraph.

Remark 3.9. There is another natural way to parametrize irreducible modules in terms of the set $\operatorname{Rev}^{\Lambda}$ of reverse-standard $\Lambda$-tableaux from 92.5 . To see this alternative parametrization, note for $A \in \operatorname{Rev}^{\Lambda}$ (and bounded-below $I$ ) that the standard module $M(A)$ has irreducible socle isomorphic to $L\left(A^{\downarrow}\right)$; see Lemma 3.3 and (2.33). Using this and arguing exactly like in the proof of Theorem 3.8, it follows (for arbitrary $I$ ) that the Specht module $S(A)$ has irreducible socle denoted $\widetilde{D}(A)$ for each $A \in \operatorname{Rev}^{\Lambda}$. Moreover

$$
\widetilde{D}(A) \cong D\left(A^{\downarrow}\right) \text {. }
$$

Applying the natural duality and recalling (3.25)-(3.26), we deduce that the module $\widetilde{S}(A) \cong S(A)^{\circledast}$ has irreducible head $\widetilde{D}(A)$ for each $A \in \operatorname{Rev}^{\Lambda}$, and the irreducible modules $\left\{\widetilde{D}(A) \mid A \in \operatorname{Rev}^{\Lambda}\right\}$ give a complete set of pairwise inequivalent irreducible $H^{\Lambda}$-modules.

For each $A \in \operatorname{Std}^{\Lambda}$, let $Y(A)$ denote the projective cover of $D(A)$; if $I$ is bounded-below then we can take $Y(A):=\pi(P(A))$ according to Theorem 3.7(ii). Then $\left\{[D(A)] \mid A \in \operatorname{Std}^{\Lambda}\right\}$ and $\left\{[Y(A)] \mid A \in \operatorname{Std}^{\Lambda}\right\}$ give canonical bases for the Grothendieck groups $\left[\operatorname{Rep}\left(H^{\Lambda}\right)\right]$ and $\left[\operatorname{Proj}\left(H^{\Lambda}\right)\right]$, respectively. These two bases are dual to each other under the Cartan pairing from (3.15). In the statement of the following theorem, the index set $I$ is arbitrary.

Theorem 3.10. Identify $\left[\operatorname{Rep}\left(H^{\Lambda}\right)\right]$ (resp. $\left.\left[\operatorname{Proj}\left(H^{\Lambda}\right)\right]\right)$ with the costandard form $V(\Lambda)_{\mathbb{Z}}^{*}$ (resp. the standard form $\left.V(\Lambda)_{\mathbb{Z}}\right)$ for the irreducible $\mathfrak{g l}_{I_{+}}(\mathbb{C})$-module of highest weight $\Lambda \in P_{+}$by identifying $[D(A)]$ with the dual-canonical basis vector $D_{A}$ (resp. $[Y(A)]$ with the canonical basis vector $P_{A}$ ) for each $A \in \operatorname{Std}^{\Lambda}$.

(i) The map $\left[\operatorname{Proj}\left(H^{\Lambda}\right)\right] \rightarrow\left[\operatorname{Rep}\left(H^{\Lambda}\right)\right]$ induced by the natural embedding of $\operatorname{Proj}\left(H^{\Lambda}\right)$ as a full subcategory $\operatorname{Rep}\left(H^{\Lambda}\right)$ is identified with the inclusion $V(\Lambda)_{\mathbb{Z}} \hookrightarrow V(\Lambda)_{\mathbb{Z}}^{*}$. In particular, this map is injective.

(ii) We have that $[S(A)]=S_{A}$ for each $A \in \mathrm{Col}^{\Lambda}$. In particular, the isomorphism classes $\left\{[S(A)] \mid A \in \operatorname{Std}^{\Lambda}\right\}$ give a basis for $\left[\operatorname{Rep}\left(H^{\Lambda}\right)\right]$ which coincides with the standard monomial basis for $V(\Lambda)_{\mathbb{Z}}^{*}$. 
(iii) For each $B \in \mathrm{Col}^{\Lambda}$ we have that

$$
[S(B)]=\sum_{A \in \operatorname{Std}^{\Lambda}} d_{A, B}(1)[D(A)]
$$

where $d_{A, B}(1)$ is the coefficient of $M_{B}$ when the canonical basis element $P_{A}$ is expanded in terms of the monomial basis of $F(\Lambda)_{\mathbb{Z}}$; see (2.16).

(iv) The Cartan pairing from (3.15) is identified with the Shapovalov pairing $\langle.,\rangle:. V(\Lambda)_{\mathbb{Z}} \times V(\Lambda)_{\mathbb{Z}}^{*} \rightarrow \mathbb{Z}$.

(v) The endomorphisms of $\left[\operatorname{Rep}\left(H^{\Lambda}\right)\right]$ and $\left[\operatorname{Proj}\left(H^{\Lambda}\right)\right]$ induced by the exact functors $e_{i}$ and $f_{i}$ from (3.19) coincide with the actions of the Chevalley generators of $U_{\mathbb{Z}}$.

(vi) For $A \in \operatorname{Std}^{\Lambda}$ and $i \in I$, the module $e_{i}\left(D(A)\right.$ ) (resp. $f_{i}(D(A))$ ) is non-zero if and only if $\tilde{e}_{i}(A) \neq \odot$ (resp. $\left.\tilde{f}_{i}(A) \neq \odot\right)$, in whch case it is a self-dual indecomposable module with irreducible socle and head isomorphic to $D\left(\tilde{e}_{i}(A)\right)$ (resp. $D\left(\tilde{f}_{i}(A)\right)$ ).

Proof. It suffices to prove the theorem in the special case that $I$ is boundedbelow. Assuming that is the case from now on, we first claim that the following diagram of $\mathbb{Z}$-linear maps commutes:

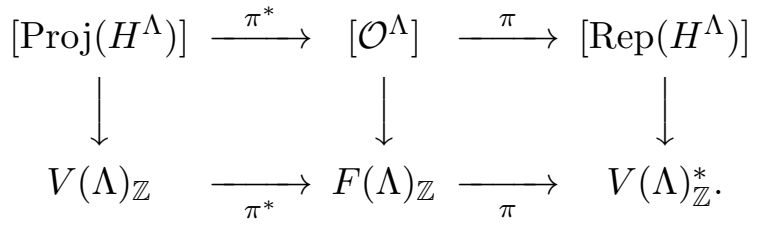

Here the top maps $\pi$ and $\pi^{*}$ are induced by the Schur functors from (3.30)(3.31). The bottom $\pi^{*}$ is the natural inclusion and the bottom $\pi$ is induced by the projection from (2.9) . The middle vertical map is the identification from Theorem 3.1 which sends $[L(A)]$ to $L_{A}$ and $[P(A)]$ to $P_{A}$ for each $A \in \mathrm{Col}^{\Lambda}$. The left (resp. right) hand vertical hand map is the identification from the statement of this theorem which maps $[Y(A)]$ to $P_{A}$ (resp. $[D(A)]$ to $\left.D_{A}\right)$ for $A \in \operatorname{Std}^{\Lambda}$. To check that the right hand square commutes, it suffices to check it commutes on $[L(A)]$ for each $A \in \mathrm{Col}^{\Lambda}$. This follows because the top map sends $[L(A)]$ to $[D(A)]$ if $A \in \operatorname{Std}^{\Lambda}$ and to zero otherwise by Theorem 3.7, while the bottom map sends $L_{A}$ to $D_{A}$ if $A \in \operatorname{Std}^{\Lambda}$ and to zero otherwise by Theorem 2.2. To check the left hand square commutes we check it on $[Y(A)]$ for each $A \in \operatorname{Std}^{\Lambda}$; this follows because by Theorem 3.7(ii) we have that $\left[\pi^{*}(Y(A))\right]=[P(A)]$. This establishes the claim.

The composite map along the top of the above diagram maps $[Y(A)]$ to $[Y(A)]$ by Theorem 3.7(ii). Hence it is exactly the map induced by the embedding of $\operatorname{Proj}\left(H^{\Lambda}\right)$ into $\operatorname{Rep}\left(H^{\Lambda}\right)$. The composite map along the bottom of the above diagram is the natural inclusion of $V(\Lambda)_{\mathbb{Z}}$ into $V(\Lambda)_{\mathbb{Z}}^{*}$. This establishes (i). Next we compute the image of $[M(A)]$ for $A \in \mathrm{Col}^{\Lambda}$ going both ways around the right hand square in the above diagram. The top map $\pi$ sends $[M(A)]$ to $[S(A)]$ by Theorem $[3.7$ (iii), while the left and bottom maps send $[M(A)]$ to $M_{A}$ then to $S_{A}$. Hence $[S(A)]$ is identified with $S_{A}$. Recalling the standard monomial basis theorem from (2.10) this proves (ii). Also (iii) now follows from (2.21) and (2.36). For (iv) we just note that $\left\{[Y(A)] \mid A \in \operatorname{Std}^{\Lambda}\right\}$ 
and $\left\{\left[D(A) \mid A \in \operatorname{Std}^{\Lambda}\right\}\right.$ are dual bases under the Cartan pairing. So we are done immediately as $\left\{P_{A} \mid A \in \operatorname{Std}^{\Lambda}\right\}$ and $\left\{D_{A} \mid A \in \operatorname{Std}^{\Lambda}\right\}$ are dual bases under the Shapovalov form according to Theorem 2.7. Now $(\mathrm{v})$ for $f_{i}$ follows from Theorem 3.1(iii) and Theorem 3.7(iv). We then get it for $e_{i}$ too using (iv) and adjointness.

Finally for (vi), it is enough to check it in the case of $f_{i}$, since it then follows easily for $e_{i}$ too by an adjointness argument. Since $f_{i}$ commutes with duality, we know at once that $f_{i} D(A)$ is self-dual. It remains to compute its head. So we need to compute $\operatorname{Hom}_{H^{\Lambda}}\left(f_{i} D(A), D(B)\right)$ for $A, B \in \operatorname{Std}^{\Lambda}$. By Theorem 3.7(v), $f_{i} D(A) \cong \pi\left(f_{i} L(A)\right)$. The modules $M:=f_{i} L(A)$ and $N:=L(B)$ satisfy the conditions from Theorem 3.7(vi) thanks to Theorem 3.1(vi). Hence we get that

$$
\operatorname{Hom}_{H^{\Lambda}}\left(f_{i} D(A), D(B)\right) \cong \operatorname{Hom}_{\mathfrak{g}}\left(f_{i} L(A), L(B)\right) .
$$

By Theorem 3.1 (vi) this is zero unless $\tilde{f}_{i}(A)=B$, when it is one dimensional. This implies (vi).

Remark 3.11. We stress that only the proof not the statement of Theorem 3.10 should be regarded as new. For example, as we said in the introduction, many parts of the theorem can be deduced from the generic case of Ariki's original theorem from [A1 using BK4, Corollary 2] and AMR, §6]. Some aspects of the theorem also appear in Grojnowski's paper G2] see [K. Theorems 9.5.1, 10.3.5]. More general results than (ii) and (vi) have been proved in the root of unity case by Jacon in [J] and Ariki in [A2], respectively.

3.7. "Tensoring with sign". In this subsection we assume that $I=\mathbb{Z}$. There is an obvious $\mathbb{Z}$-linear map $t: P \rightarrow P$ mapping $\Lambda_{i}$ to $\Lambda_{-i}$ and $\alpha_{i}$ to $\alpha_{-i}$ for each $i \in \mathbb{Z}$. We let

$$
\Lambda^{t}:=\Lambda_{-m_{l}}+\cdots+\Lambda_{-m_{1}}
$$

denote the image of $\Lambda$ under this map; note that $-m_{l} \geq \cdots \geq-m_{1}$.

We are interested in the sign automorphism

$$
\sigma: H_{d} \rightarrow H_{d}, \quad s_{r} \mapsto-s_{r}, x_{r} \mapsto-x_{r} .
$$

For an $H_{d}$-module $M$, we write $\sigma^{*}(M)$ for the $H_{d}$-module obtained from $M$ by twisting the action by this automorphism $\sigma$. Recalling (3.23), we note for any $\mathbb{C} S_{d}$-module $M$ that

$$
\sigma^{*}\left(\operatorname{ev}_{m}^{*}(M)\right) \cong \mathrm{ev}_{-m}^{*}(M \otimes \operatorname{sgn})
$$

where $M \otimes \operatorname{sgn}$ denotes the $\mathbb{C} S_{d}$-module obtained by tensoring $M$ with the one dimensional sign representation. Moreover,

$$
\begin{aligned}
\sigma^{*}\left(M^{\circledast}\right) & \cong \sigma^{*}(M)^{\circledast}, \\
\sigma^{*}(M \circ N) & \cong \sigma^{*}(M) \circ \sigma^{*}(N),
\end{aligned}
$$

where $\circ$ is the induction product from (3.22).

Suppose also that we are given $\alpha \in Q_{+}$of height $d$. Recalling (3.7), let

$$
H_{d}^{\boldsymbol{m}^{t}}:=H_{d} /\left\langle\left(x_{1}+m_{l}\right) \cdots\left(x_{1}+m_{1}\right)\right\rangle .
$$

Notice that the automorphism $\sigma$ factors through the quotients to induce an isomorphism $H_{d}^{\boldsymbol{m}} \stackrel{\sim}{\rightarrow} H_{d}^{\boldsymbol{m}^{t}}$. This isomorphism maps the idempotent $e\left(i_{1}, \ldots, i_{d}\right)$ 
to the idempotent $e\left(-i_{1}, \ldots,-i_{d}\right)$, hence it sends the block idempotent $e_{\alpha}$ to $e_{\alpha^{t}}$. This establishes that $\sigma$ induces an isomorphism

$$
\sigma: H_{\alpha}^{\Lambda} \stackrel{\sim}{\rightarrow} H_{\alpha^{t}}^{\Lambda^{t}}
$$

If $M$ is an $H_{\alpha^{t}}^{\Lambda^{t}}$-module then $\sigma^{*}(M)$ is naturally an $H_{\alpha}^{\Lambda}$-module.

We define the transpose $\lambda^{t}$ of an $l$-multipartition $\lambda=\left(\lambda^{(1)}, \ldots, \lambda^{(l)}\right)$ to be the $l$-multipartition $\left(\left(\lambda^{(l)}\right)^{t}, \ldots,\left(\lambda^{(1)}\right)^{t}\right)$, where $\left(\lambda^{(r)}\right)^{t}$ is the usual transpose of the partition $\lambda^{(r)}$. There is a bijection

$$
\mathrm{Col}_{\alpha}^{\Lambda} \stackrel{\sim}{\rightarrow} \operatorname{Col}_{\alpha^{t}}^{\Lambda^{t}}, \quad A \mapsto A^{t}
$$

where for a $\Lambda$-tableau $A$, we write $A^{t}$ for the unique $\Lambda^{t}$-tableau that satsifies $\lambda\left(A^{t}\right)=\lambda(A)^{t}$, recalling the notation (2.50). We stress that this depends implicitly on the fixed choice of $\Lambda$.

Lemma 3.12. The bijection (3.40) restricts to a bijection $\operatorname{Rev}_{\alpha}^{\Lambda} \stackrel{\sim}{\rightarrow} \operatorname{Std}_{\alpha^{t}}^{\Lambda^{t}}$.

Proof. Using (2.52)-(2.53) and the definitions, this reduces to checking for partitions $\lambda$ and $\mu$ and an integer $m \geq 0$ that $\lambda_{j}+m \geq \mu_{j}$ for all $j \geq 1$ if and only if $\mu_{j+m}^{t} \leq \lambda_{j}^{t}$ for all $j \geq 1$. This is an easy combinatorial exercise.

Lemma 3.13. For $A \in \operatorname{Col}_{\alpha}^{\Lambda}$, we have that $\sigma^{*}\left(S\left(A^{t}\right)\right) \cong \widetilde{S}(A) \cong S(A)^{\circledast}$.

Proof. Recalling the definition (3.24), we have by (3.35) and (3.37) that

$$
\begin{aligned}
\sigma^{*}\left(S\left(A^{t}\right)\right) & =\sigma^{*}\left(\operatorname{ev}_{-m_{l}}^{*}\left(S\left(\left(\lambda^{(l)}\right)^{t}\right)\right) \circ \cdots \circ \mathrm{ev}_{-m_{1}}^{*}\left(S\left(\left(\lambda^{(1)}\right)^{t}\right)\right)\right) \\
& \cong \operatorname{ev}_{m_{l}}^{*}\left(S\left(\left(\lambda^{(l)}\right)^{t}\right) \otimes \operatorname{sgn}\right) \circ \cdots \circ \mathrm{ev}_{m_{1}}^{*}\left(S\left(\left(\lambda^{(1)}\right)^{t}\right) \otimes \operatorname{sgn}\right) .
\end{aligned}
$$

Since $S\left(\mu^{t}\right) \otimes \operatorname{sgn} \cong S(\mu)$ for ordinary representations of the symmetric group, this equals $\widetilde{S}(A)$ according to the definition (3.25), which is isomorphic to $S(A)^{\circledast}$ by (3.26).

Now we can describe what happens when we twist irreducible $H_{\alpha^{t}}^{\Lambda^{t}}$-modules by the automorphism $\sigma$. In particular, this theorem gives a first explanation for the alternate parametrization of irreducible modules mentioned in Remark 3.9.

Theorem 3.14. For $A \in \operatorname{Rev}_{\alpha}^{\Lambda}$, we have that $\sigma^{*}\left(D\left(A^{t}\right)\right) \cong \widetilde{D}(A) \cong D\left(A^{\downarrow}\right)$.

Proof. By Theorem 3.8 and Lemma 3.12, $D\left(A^{t}\right)$ is the irreducible head of $S\left(A^{t}\right)$. Hence $\sigma^{*}\left(D\left(A^{t}\right)\right)$ is the irreducible head of $\sigma^{*}\left(S\left(A^{t}\right)\right)$, which is isomorphic to $\widetilde{S}(A)$ according to Lemma 3.13. Now use Remark 3.9.

\section{Young modules And TILTING MOdules}

In this section we prove a number of additional results which complete the Schur-Weyl duality picture from BK3]. The results in $\S \$ 4.34 .4$ should be compared with Mathas' results from [M]. 
4.1. Schur functors commute with duality. Recall the dualities $\circledast$ on $\mathcal{O}^{\Lambda}$ and on $\operatorname{Rep}\left(H^{\Lambda}\right)$ defined in 33.1 and 33.3 , respectively. We want to prove that these two dualities are intertwined by the Schur functor $\pi$ from (3.30). Recall to start with from [BK3, Theorem A.2] that $H_{d}^{m}$ is a symmetric algebra with symmetrizing form $\tau: H_{d}^{m} \rightarrow \mathbb{C}$ defined by picking out the $x_{1}^{l-1} \cdots x_{d}^{l-1}$ coefficient of a vector when expanded in terms of the basis (3.8). This restricts to a symmetrizing form on each $H_{\alpha}^{\Lambda}$ for $\alpha \in Q_{+}$with $\mathrm{ht}(\alpha)=d$. Hence each $H_{\alpha}^{\Lambda}$ is also a symmetric algebra.

As well as the duality $\circledast$ on $\operatorname{Rep}\left(H_{\alpha}^{\Lambda}\right)$, there is another duality \# mapping a left module $M$ to

$$
M^{\#}:=\operatorname{Hom}_{H_{\alpha}^{\Lambda}}\left(M, H_{\alpha}^{\Lambda}\right)
$$

viewed as a left $H_{\alpha}^{\Lambda}$-module with action $(h f)(m)=f(m) h^{*}$ for $h \in H_{\alpha}^{\Lambda}, m \in M$ and $f: M \rightarrow H_{\alpha}^{\Lambda}$; here $h^{*}$ denotes the image of $h$ under the anti-automorphism from (3.20). By general principles, the fact that $H_{\alpha}^{\Lambda}$ is symmetric implies that this duality is equivalent to the duality $\circledast$.

Lemma 4.1. For any finite dimensional left $H_{\alpha}^{\Lambda}$-module $M$, there is a natural $H_{\alpha}^{\Lambda}$-module isomorphism $M^{\#} \stackrel{\sim}{\rightarrow} M^{\circledast}, f \mapsto \tau \circ f$.

Proof. See [R, Theorem 3.1].

Now fix $\alpha \in Q_{+}$of height $d$ and identify $H_{\alpha}^{\Lambda}$ with $\operatorname{End}_{\mathfrak{g}}\left(T_{\alpha}^{\Lambda}\right)^{\text {op }}$ according to Theorem 3.6 .

Lemma 4.2. There is a non-degenerate symmetric bilinear form (., .) on $T_{\alpha}^{\Lambda}$ such that $(x v h, w)=\left(v, x^{*} w h^{*}\right)$ for all $v, w \in T_{\alpha}^{\Lambda}, x \in \mathfrak{g}$ and $h \in H_{\alpha}^{\Lambda}$ (recall $x^{*}$ denotes the transpose of the matrix $x)$.

Proof. Recalling that $T_{\alpha}^{\Lambda}$ is a block of $P\left(A^{\Lambda}\right) \otimes T_{d}$, it suffices to construct such a non-degenerate form on all of $P\left(A^{\Lambda}\right) \otimes T_{d}$. As $P\left(A^{\Lambda}\right)$ is irreducible, it admits a non-degenerate symmetric bilinear form $(.,$.$) such that (x v, w)=\left(v, x^{*} w\right)$ for each $x \in \mathfrak{g}$ and $v, w \in P\left(A^{\Lambda}\right)$. There is also a non-degenerate symmetric bilinear form on $T_{d}$ with respect to which the monomials in the standard basis of the natural $\mathfrak{g}$-module are orthonormal. The product of these forms gives us a non-degenerate symmetric bilinear form on $P\left(A^{\Lambda}\right) \otimes T_{d}$ such that $(x v, w)=$ $\left(v, x^{*} w\right)$ again. The fact that $(v h, w)=\left(v, w h^{*}\right)$ for each $h \in H_{d}$ is clear for the action of each $s_{r}$, and follows for the action of $x_{1}$ by the definition of the form as a product, recalling $x_{1}$ acts as multiplication by $\Omega$.

For the next two lemmas, we define another functor

$$
\delta:=\operatorname{Hom}_{\mathfrak{g}}\left(?, T_{\alpha}^{\Lambda}\right): \mathcal{O}_{\alpha}^{\Lambda} \rightarrow \operatorname{Rep}\left(H_{\alpha}^{\Lambda}\right),
$$

with $h \in H_{\alpha}^{\Lambda}$ acting on $f \in \delta(M)=\operatorname{Hom}_{\mathfrak{g}}\left(M, T_{\alpha}^{\Lambda}\right)$ by the rule $(h f)(m)=$ $f(m) h^{*}$.

Lemma 4.3. There is an isomorphism $\delta \circ \pi^{*} \cong \#$ as endofunctors of $\operatorname{Rep}\left(H_{\alpha}^{\Lambda}\right)$.

Proof. There are natural isomorphisms

$$
\begin{aligned}
\delta \circ \pi^{*}(M) & =\operatorname{Hom}_{\mathfrak{g}}\left(T_{\alpha}^{\Lambda} \otimes_{H_{\alpha}^{\Lambda}} M, T_{\alpha}^{\Lambda}\right) \\
& \cong \operatorname{Hom}_{H_{\alpha}^{\Lambda}}\left(M, \operatorname{End}_{\mathfrak{g}}\left(T_{\alpha}^{\Lambda}\right)\right) \cong \operatorname{Hom}_{H_{\alpha}^{\Lambda}}\left(M, H_{\alpha}^{\Lambda}\right)=M^{\#},
\end{aligned}
$$


for any $M \in \operatorname{Rep}\left(H_{\alpha}^{\Lambda}\right)$.

Lemma 4.4. There is an isomorphism $\delta \cong \pi \circ \circledast$ of functors from $\mathcal{O}_{\alpha}^{\Lambda}$ to $\operatorname{Rep}\left(H_{\alpha}^{\Lambda}\right)$.

Proof. Define a natural homomorphism $\delta(M) \rightarrow \pi\left(M^{\circledast}\right)$ for any $M \in \mathcal{O}_{\alpha}^{\Lambda}$ by mapping $f \in \delta(M)=\operatorname{Hom}_{\mathfrak{g}}\left(M, T_{\alpha}^{\Lambda}\right)$ to $\hat{f} \in \pi\left(M^{\circledast}\right)=\operatorname{Hom}_{\mathfrak{g}}\left(T_{\alpha}^{\Lambda}, M^{\circledast}\right)$, where $\hat{f}(t)(m)=(t, f(m))$ for $t \in T_{\alpha}^{\Lambda}$ and $m \in M$. Here, (.,.) is the bilinear form from Lemma 4.2. The non-degeneracy of this form gives easily that our map is injective. It is an isomorphism because $\delta(M)$ and $\pi\left(M^{\circledast}\right)$ both have the same dimension. To see that, note that $M$ and $M^{\circledast}$ have all the same composition multiplicities, and $T_{\alpha}^{\Lambda}$ is a direct sum of prinjective indecomposable modules, each of which is both the projective cover and the injective hull of the same irreducible module.

Theorem 4.5. There is an isomorphism $\pi \circ \circledast \cong \circledast \circ \pi$ of functors from $\mathcal{O}_{\alpha}^{\Lambda}$ to $\operatorname{Rep}\left(H_{\alpha}^{\Lambda}\right)$.

Proof. In view of Lemma 4.1, it suffices to show that $\pi \cong \# \circ \pi \circ \circledast$. By Lemmas 4.3 and 4.4, we have that

$$
\# \circ \pi \circ \circledast \cong \# \circ \delta \cong \delta \circ \pi^{*} \circ \delta \cong \pi \circ \circledast \circ \pi^{*} \circ \delta .
$$

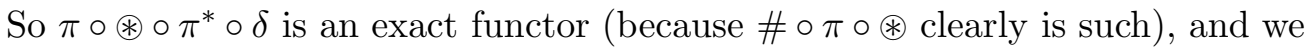
need to show that $\pi \cong \pi \circ \circledast \circ \pi^{*} \circ \delta$.

Let us first construct a natural $\mathfrak{g}$-module homomorphism

$$
\theta_{M}: M \rightarrow \circledast \circ \pi^{*} \circ \delta(M)=\left[T_{\alpha}^{\Lambda} \otimes_{H_{\alpha}^{\Lambda}} \operatorname{Hom}_{\mathfrak{g}}\left(M, T_{\alpha}^{\Lambda}\right)\right]^{\circledast}
$$

for every $M \in \mathcal{O}_{\alpha}^{\Lambda}$, by letting $\theta_{M}(m)(t \otimes f):=(t, f(m))$ for $m \in M, t \in T_{\alpha}^{\Lambda}$ and $f \in \operatorname{Hom}_{\mathfrak{g}}\left(M, T_{\alpha}^{\Lambda}\right)$. Here $(.,$.$) is the bilinear form from Lemma 4.2$ again. To see that $\theta_{M}$ is well-defined, note that

$$
\theta_{M}(m)(t h \otimes f)=(t h, f(m))=\left(t, f(m) h^{*}\right)=\theta_{M}(m)(t \otimes h f) .
$$

To see that $\theta_{M}$ is a $\mathfrak{g}$-module homomorphism, note that

$$
\theta_{M}(x m)(t \otimes f)=(t, f(x m))=(t, x f(m))=\left(x^{*} t, f(m)\right)=\left(x \theta_{M}(m)\right)(t \otimes f) .
$$

Observe finally that $\theta_{M}$ is an isomorphism in the special case that $M=T_{\alpha}^{\Lambda}$. Indeed, in this case, $T_{\alpha}^{\Lambda} \otimes_{H_{\alpha}^{\Lambda}} \operatorname{Hom}_{\mathfrak{g}}\left(M, T_{\alpha}^{\Lambda}\right)$ can be identified simply with $T_{\alpha}^{\Lambda}$, and $\theta_{M}$ is the isomorphism $T_{\alpha}^{\Lambda} \stackrel{\sim}{\rightarrow}\left(T_{\alpha}^{\Lambda}\right)^{\circledast}$ induced by the non-degenerate bilinear form $(.,$.$) .$

Applying the functor $\pi$ to the homomorphism $\theta_{M}$ from the previous paragraph, we obtain a natural $H_{\alpha}^{\Lambda}$-module homomorphism

$$
\eta_{M}: \pi(M) \rightarrow \pi \circ \circledast \circ \pi^{*} \circ \delta(M), \quad f \mapsto \theta_{M} \circ f
$$

for $M \in \mathcal{O}_{\alpha}^{\Lambda}$. We have now defined a natural transformation $\eta: \pi \rightarrow \pi \circ \circledast \circ \pi^{*} \circ \delta$ between two exact functors. To complete the proof of the theorem, we need to show that this natural transformation is an isomorphism. Taking $M=T_{\alpha}^{\Lambda}$, we get that $\eta_{M}$ is an isomorphism, because $\theta_{M}$ is already an isomorphism in that case by the last statement of the previous paragraph. Hence, by Lemma 3.5 and naturality, $\eta_{M}$ is an isomorphism whenever $M$ is a prinjective module. Moreover 
by the proof of [BK3, Theorem 6.10], every projective module $M \in \mathcal{O}_{\alpha}^{\Lambda}$ has a two step resolution $0 \rightarrow M \rightarrow I_{1} \rightarrow I_{2}$ where $I_{1}$ and $I_{2}$ are prinjective modules. Using this and the five lemma, we get that $\eta_{M}$ is an isomorphism whenever $M$ is a projective module in $\mathcal{O}_{\alpha}^{\Lambda}$. Finally for an arbitrary $M$ we take a two step projective resolution $P_{2} \rightarrow P_{1} \rightarrow M \rightarrow 0$ and apply the five lemma once more.

4.2. Young modules and the double centralizer property. We next introduce another important family of $H_{\alpha}^{\Lambda}$-modules, the so-called Young modules $Y(A)$ for $A \in \operatorname{Col}_{\alpha}^{\Lambda}$. For $I$ bounded-below, these are simply the images of the projective indecomposable modules $P(A)$ in $\mathcal{O}_{\alpha}^{\Lambda}$ under the Schur functor $\pi$. We will give a more intrinsic definition which is valid for arbitrary $I$.

So assume that $I$ is arbitrary and $\Lambda$ and $\alpha$ are fixed as usual. Given $A \in \mathrm{Col}_{\alpha}^{\Lambda}$, set $\lambda=\left(\lambda^{(1)}, \ldots, \lambda^{(l)}\right):=\lambda(A)$ and $d_{i}:=\left|\lambda^{(i)}\right|$ for each $i=1, \ldots, l$. Note that $d=d_{1}+\cdots+d_{l}$ is the height of $\alpha$. Let $S_{\lambda^{(i)}}$ denote the parabolic subgroup $S_{\lambda_{1}^{(i)}} \times S_{\lambda_{2}^{(i)}} \times \cdots$ of the symmetric group $S_{d_{i}}$, so that $S_{\lambda^{(1)}} \times \cdots \times S_{\lambda^{(l)}}$ is a parabolic subgroup of $S_{d}$. Define $X(A)$ to be the left ideal of $H_{\alpha}^{\Lambda}$ generated by the element

$$
\prod_{i=2}^{l} \prod_{j=1}^{d_{1}+\cdots+d_{i-1}}\left(x_{j}-m_{i}\right) \cdot \sum_{w \in S_{\lambda}(1) \times \cdots \times S_{\lambda}(l)} w .
$$

We call $X(A)$ the reduced permutation module because it is a block-wise version of (the degenerate analogue of) the permutation module of Dipper, James and Mathas [DJM]. More precisely, the Dipper-James-Mathas permutation module is the left ideal of $H_{d}^{m}$ generated by the element (4.3), and then our $X(A)$ is obtained from that by multiplying by the block idempotent $e_{\alpha}$.

To connect the reduced permutation module $X(A)$ to parabolic category $\mathcal{O}$, assume in this paragraph that $I$ is bounded-below, so that the Schur functor $\pi$ from (3.30) is defined. Recall the divided power module $Z(A)$ from [BK3, (4.7)]:

$$
Z(A):=U(\mathfrak{g}) \otimes_{U(\mathfrak{p})}\left[I^{\Lambda} \otimes Z^{\lambda^{(1)}}\left(V_{1}\right) \otimes \cdots \otimes Z^{\lambda^{(l)}}\left(V_{l}\right)\right] .
$$

Some explanations are needed here. First $\mathfrak{p}$ is the standard parabolic subalgebra of $\mathfrak{g}$ with Levi factor $\mathfrak{g l}_{n_{1}}(\mathbb{C}) \oplus \cdots \oplus \mathfrak{g l}_{n_{l}}(\mathbb{C})$ as in 33.1 . Then $I^{\Lambda}$ denotes the one dimensional $\mathfrak{p}$-module associated to the weight

$$
\sum_{i=1}^{l}\left(n_{1}+\cdots+n_{i-1}+m_{i}\right)\left(\varepsilon_{n_{1}+\cdots+n_{i-1}+1}+\cdots+\varepsilon_{n_{1}+\cdots+n_{i}}\right) .
$$

Next $V_{i}$ denotes the $\mathfrak{p}$-submodule of the natural $\mathfrak{g}$-module of column vectors spanned by the first $n_{1}+\cdots+n_{i}$ of the standard basis vectors. Finally for a vector space $E$ and a partition $\mu$ of $n$ we write $Z^{\mu}(E)$ for the submodule of $E^{\otimes n}$ consisting of all tensors that are invariant with respect to the natural action of the parabolic subgroup $S_{\mu}$ of $S_{n}$. According to [BK3, Theorem 4.14], we have that

$$
Z(A)=P(A) \oplus(*)
$$

where $(*)$ is a direct sum of $P(B)$ 's for $B \in \mathrm{Col}^{\Lambda}$ that are higher than $A$ in the sense that $\lambda(B)>\lambda(A)$ in the dominance order on multipartitions, i.e. the 
diagram of $\lambda(B)$ is obtained from that of $\lambda(A)$ by moving boxes up. We also need the following key isomorphism which is established in [BK3, Theorem 6.9]: for every $A \in \mathrm{Col}_{\alpha}^{\Lambda}$ there is an isomorphism

$$
X(A) \cong \operatorname{Hom}_{\mathfrak{g}}\left(T_{\alpha}^{\Lambda}, Z(A)\right)
$$

of $H_{\alpha}^{\Lambda}$-modules. Now we can formulate the following theorem which gives the intrinsic definition of Young modules.

Theorem 4.6. For any index set $I$ and $A \in \mathrm{Col}_{\alpha}^{\Lambda}$, there exists a unique (up to isomorphism) indecomposable $H_{\alpha}^{\Lambda}$-module $Y(A)$ such that

$$
X(A)=Y(A) \oplus(\dagger)
$$

where $(\dagger)$ denotes a direct sum of $Y(B)$ 's for $B \in \mathrm{Col}_{\alpha}^{\Lambda}$ with $\lambda(B)>\lambda(A)$ in the dominance order on multipartitions. Moreover:

(i) If $A \in \operatorname{Std}_{\alpha}^{\Lambda}$ then $Y(A)$ coincides with the projective cover of the irreducible $H_{\alpha}^{\Lambda}$-module $D(A)$ from Theorem 3.8 .

(ii) If $I$ is bounded-below then $Y(A) \cong \pi(P(A))$ for each $A \in \mathrm{Col}_{\alpha}^{\Lambda}$.

Proof. Since $X(A)$ does not depend on the particular choice of $I$, we may as well assume that $I$ is bounded-below. In that case we define $Y(A)$ to be the $H_{\alpha}^{\Lambda}$-module $\pi(P(A))$ for each $A \in \mathrm{Col}_{\alpha}^{\Lambda}$, so that (ii) is automatic. By Theorem 3.7(ii) this is the projective cover of $D(A)$ whenever $A \in \operatorname{Std}_{\alpha}^{\Lambda}$, giving (i). Moreover by (4.6) we have that $X(A)=e_{\alpha} \pi(Z(A))$, hence by (4.5) we see that

$$
X(A)=Y(A)+(\dagger)
$$

where $(\dagger)$ consists of higher $Y(B)$ 's as in the statement of the theorem. It remains to observe that $Y(A)$ is indecomposable. Since $P(A)$ is projective and indecomposable, we get by Theorem 3.7(vi) and Fitting's lemma that

$$
\operatorname{End}_{H_{\alpha}^{\Lambda}}(Y(A)) \cong \operatorname{End}_{\mathfrak{g}}(P(A))
$$

is a local ring. Hence $Y(A)$ is indecomposable as required.

The main reason Young modules are important is the following theorem, which we view as a "double centralizer property". By a Young generator for $H_{\alpha}^{\Lambda}$, we mean a finite dimensional module that is isomorphic to a direct sum of the Young modules $Y(A)$ for $A \in \mathrm{Col}_{\alpha}^{\Lambda}$, with each appearing at least once.

Theorem 4.7. Assume that $I$ is bounded-below and $\alpha \in Q_{+}$. Let $Y$ be a Young generator for $H_{\alpha}^{\Lambda}$. Then the category of finite dimensional left modules over the endomorphism algebra $\operatorname{End}_{H_{\alpha}^{\Lambda}}(Y)^{\mathrm{op}}$ is equivalent to the category $\mathcal{O}_{\alpha}^{\Lambda}$.

Proof. By Theorem 4.6(ii), there is a projective generator $P$ for $\mathcal{O}_{\alpha}^{\Lambda}$ such that $Y \cong \pi(P)$. By general principles, the category of finite dimensional left modules over $\operatorname{End}_{\mathfrak{g}}(P)^{\text {op }}$ is equivalent to $\mathcal{O}_{\alpha}^{\Lambda}$. By Theorem 3.7(vi) the Schur functor $\pi$ is fully faithful on projectives, so $\pi$ defines an algebra isomorphism

$$
\operatorname{End}_{\mathfrak{g}}(P)^{\mathrm{op}} \cong \operatorname{End}_{H_{\alpha}^{\Lambda}}(Y)^{\mathrm{op}} \text {. }
$$

The theorem follows. 
Corollary 4.8. If $Y$ is a Young generator for $H_{\alpha}^{\Lambda}$ then the finite dimensional algebra $\operatorname{End}_{H_{\alpha}^{\Lambda}}(Y)^{\mathrm{op}}$ admits a unique (up to automorphism) grading with respect to which it is a Koszul algebra.

Proof. This follows from the theorem by general results of Beilinson, Ginzburg and Soergel [BGS, Theorem 1.1.3] and Backelin [B, Theorem 1.1]; see also [BGS, Corollary 2.5.2] for the unicity.

Remark 4.9. In particular the (degenerate) cyclotomic Schur algebra of Dipper, James and Mathas over the ground field $\mathbb{C}$ as defined in [AMR, §6] is a Koszul algebra. This follows from Corollary 4.8 because the blocks of the cyclotomic Schur algebra are endomorphism algebras of Young generators of corresponding $H_{\alpha}^{\Lambda}$ 's; see [BK3, Theorem 6.10].

4.3. Tilting modules and the opposite Schur-Weyl duality. We assume in this subsection that the index set $I$ is bounded-below. As well as the parabolic category $\mathcal{O}^{\Lambda}$, we also introduced the opposite parabolic category $\widetilde{\mathcal{O}}^{\Lambda}$ in 3.1. There is a precise connection between these two categories provided by Arkhipov's twisting functor associated to the longest element $w_{0}$ of the symmetric group $S_{n}$. In particular this functor is the key to the proof of the Arkhipov-Soergel reciprocity formula recorded already in (3.5).

To give some more details, let $S_{w_{0}}$ be Arkhipov's semi-regular bimodule as in $[\mathrm{S}$, Theorem 1.3], which is the $(U(\mathfrak{g}), U(\mathfrak{g}))$-bimodule associated to the longest element $w_{0}$ in the setup of [AnS, p.684]. Then Arkipov's twisting functor

$$
\mathrm{T}_{w_{0}}: \widetilde{\mathcal{O}}^{\Lambda} \rightarrow \mathcal{O}^{\Lambda}
$$

is the right exact functor defined by first tensoring with $S_{w_{0}}$ then twisting by the automorphism $\varphi_{w_{0}}: \mathfrak{g} \rightarrow \mathfrak{g}$ arising from conjugating by the longest element of the symmetric group $S_{n}$ (viewed as a permutation matrix):

$$
\mathrm{T}_{w_{0}}(M):=\varphi_{w_{0}}^{*}\left(S_{w_{0}} \otimes_{U(\mathfrak{g})} M\right) .
$$

The key properties of this functor are summarized in the following known lemma; note in particular that (3.5) follows at once from this and the selfduality of tilting modules. In the statement of the lemma, we say that an object in $\widetilde{\mathcal{O}}^{\Lambda}$ (resp. $\mathcal{O}^{\Lambda}$ ) has a standard (resp. costandard) filtration if it admits a filtration whose sections are of the form $\widetilde{M}(A)$ (resp. $\left.M(A)^{\circledast}\right)$ for $A \in \mathrm{Col}^{\Lambda}$.

Lemma 4.10. The twisting functor $\mathrm{T}_{w_{0}}$ defines an equivalence between the full subcategory of $\widetilde{\mathcal{O}}^{\Lambda}$ consisting of all modules possessing a standard filtration and the full subcategory of $\mathcal{O}^{\Lambda}$ consisting of all modules possessing a costandard filtration. Moreover for $A \in \mathrm{Col}^{\Lambda}$ we have that

(i) $\mathrm{T}_{w_{0}}(\widetilde{M}(A)) \cong M(A)^{\circledast}$;

(ii) $\mathrm{T}_{w_{0}}(\widetilde{P}(A)) \cong T(A)$.

Proof. This is a slight reformulation of [S, Theorem 6.6] in our special case.

We also need the following lemma showing that the functor $\mathrm{T}_{w_{0}}$ commutes nicely with tensoring with finite dimensional modules. 
Lemma 4.11. There is a natural $\mathfrak{g}$-module isomorphism

$$
j_{M, X}: \mathrm{T}_{w_{0}}(M \otimes X) \stackrel{\sim}{\rightarrow} \mathrm{T}_{w_{0}}(M) \otimes X
$$

for every $\mathfrak{g}$-module $M$ and every finite dimensional $\mathfrak{g}$-module $X$. This isomorphism has the following additional properties.

(i) Given also a finite dimensional $\mathfrak{g}$-module $Y$, the following diagram commutes:

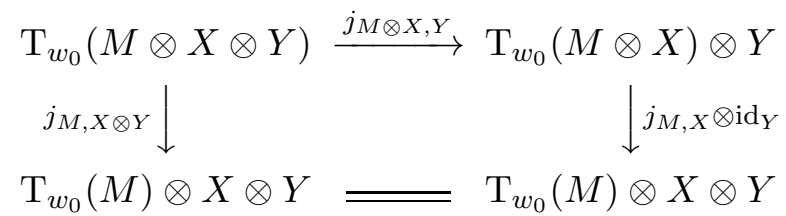

(ii) The isomorphism $j_{M, X}$ commutes with the action of $z \in Z(U(\mathfrak{g}))$ in the sense that $j_{M, X} \circ \mathrm{T}_{w_{0}}\left(\lambda_{z}\right)=\lambda_{z} \circ j_{M, X}$, where $\lambda_{z}$ denotes the $\mathfrak{g}$-module endomorphism defined by left multiplication by $z$.

Proof. All of this except for part (ii) follow at once from [AnS, Theorem 3.2]. To deduce (ii), recall from AnS that the functor $\mathrm{T}_{w_{0}}$ is a composite of functors $\mathrm{T}_{s}$ for simple reflections $s$ (taken in order corresponding to a reduced expression for $\left.w_{0}\right)$, and then the isomorphism $j_{M, X}$ above is built from analagous isomorphisms

$$
j_{M, X}^{s}: \mathrm{T}_{s}(M \otimes X) \stackrel{\sim}{\rightarrow} \mathrm{T}_{s}(M) \otimes X
$$

for each simple reflection $s$. In view of this, it suffices to show that $j_{M, X}^{s} \circ$ $\mathrm{T}_{s}\left(\lambda_{z}\right)=\lambda_{z} \circ j_{M, X}^{s}$ for each $s$. The explicit formula for $j_{M, X}^{s}$ from the proof of [AnS, Theorem 3.2] gives that

$$
j_{M, X}^{s}(u(1 \otimes(m \otimes e)))=\widetilde{\Delta}(u)((1 \otimes m) \otimes e)
$$

for $u \in U_{(s)}, m \in M$ and $e \in X$. Here, we are using the notation from [AnS]; in particular, $U_{(s)}$ is a certain Ore localization of $U$ and $\widetilde{\Delta}: U_{(s)} \rightarrow U_{(s)} \hat{\otimes} U_{(s)}$ is a certain homomorphism extending the comultiplication $\Delta: U \rightarrow U \otimes U$. Using this formula, the problem reduces to checking that

$$
j_{M, X}^{s}(u(1 \otimes z(m \otimes e)))=\widetilde{\Delta}(u) \Delta(z)((1 \otimes m) \otimes e)
$$

is equal to

$$
z j_{M, X}^{s}(u(1 \otimes(m \otimes e)))=\Delta(z) \widetilde{\Delta}(u)((1 \otimes m) \otimes e) .
$$

To see this, observe that

$$
\widetilde{\Delta}(u) \Delta(z)=\widetilde{\Delta}(u z)=\widetilde{\Delta}(z u)=\Delta(z) \widetilde{\Delta}(u)
$$

as $z$ is central.

Now we want to consider the analogue of the Schur-Weyl duality from 33.5 with the category $\mathcal{O}^{\Lambda}$ replaced by $\widetilde{\mathcal{O}}^{\Lambda}$. As in the first paragraph of $\$ 3.5$, there is a natural right action of the degenerate affine Hecke algebra $H_{d}$ on the module $\widetilde{P}\left(A^{\Lambda}\right) \otimes T_{d}$ commuting with the left action of $\mathfrak{g}$. For $\alpha \in Q_{+}$with $\mathrm{ht}(\alpha)=d$, define $\widetilde{T}_{\alpha}^{\Lambda}$ to be the projection of $\widetilde{P}\left(A^{\Lambda}\right) \otimes T_{d}$ onto the block $\widetilde{\mathcal{O}}_{\alpha}^{\Lambda}$. This is again a $\left(U(\mathfrak{g}), H_{d}\right)$-bimodule. 
Lemma 4.12. The modules $\left\{\widetilde{P}(A) \mid A \in \operatorname{Rev}_{\alpha}^{\Lambda}\right\}$ give a full set of prinjective indecomposable modules in $\widetilde{\mathcal{O}}_{\alpha}^{\Lambda}$. Moreover, $\widetilde{T}_{\alpha}^{\Lambda}$ is a prinjective generator for $\widetilde{\mathcal{O}}_{\alpha}^{\Lambda}$

Proof. Mimic the proofs of Lemmas 3.2 and 3.5 (i.e. [BK3, Theorem 4.8] and [BK3, Corollary 4.6]), using the reverse crystal structure from 92.5 instead of the usual crystal structure.

The following lemma gives the analogue of Theorem 3.6 in the twisted setup; see also [B2, Lemma 5.5] for a different approach yielding a slightly more general result.

Theorem 4.13. There is a $\mathfrak{g}$-module isomorphism $j_{\alpha}: \mathrm{T}_{w_{0}}\left(\widetilde{T}_{\alpha}^{\Lambda}\right) \stackrel{\sim}{\rightarrow} T_{\alpha}^{\Lambda}$ such that the following diagram of algebra homomorphisms commutes:

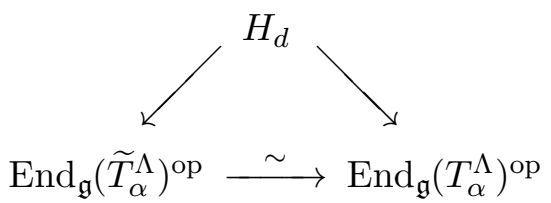

where the vertical maps come from the $H_{d^{-}}$actions on $\widetilde{T}_{\alpha}^{\Lambda}$ and on $T_{\alpha}^{\Lambda}$, respectively, and the bottom map is the algebra isomorphism $\theta \mapsto j_{\alpha} \circ \mathrm{T}_{w_{0}}(\theta) \circ j_{\alpha}^{-1}$. Hence, the action of $H_{d}$ on $\widetilde{T}_{\alpha}^{\Lambda}$ induces a canonical isomorphism between $H_{\alpha}^{\Lambda}$ and $\operatorname{End}_{\mathfrak{g}}\left(\widetilde{T}_{\alpha}^{\Lambda}\right)^{\text {op }}$.

Proof. Set $M:=\widetilde{P}\left(A^{\Lambda}\right)$ for short. Consider the isomorphism

$$
j_{M, T_{d}}: \mathrm{T}_{w_{0}}\left(M \otimes T_{d}\right) \stackrel{\sim}{\rightarrow} \mathrm{T}_{w_{0}}(M) \otimes T_{d}
$$

from Lemma 4.11. We have natural actions of $H_{d}$ on $\mathrm{T}_{w_{0}}\left(M \otimes T_{d}\right)$ and on $\mathrm{T}_{w_{0}}(M) \otimes T_{d}$; the former means the action obtained by applying the functor $\mathrm{T}_{w_{0}}$ to the natural action of $H_{d}$ on $M \otimes T_{d}$. We claim that the isomorphism $j_{M, T_{d}}$ intertwines these two actions. This is clear for the actions of each $w \in S_{d}$ by the naturality of $j$. It remains to see that $j_{M, T_{d}}$ intertwines the two actions of $x_{1}$. Note that the following diagram commutes by a special case of Lemma 4.11(i):

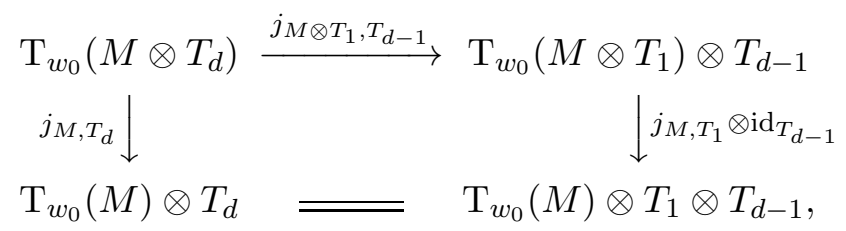

Using this, our problem reduces to checking that $j_{M, T_{1}}$ intertwines the two actions of $x_{1}$, i.e. we may assume that $d=1$. In that case, $x_{1}$ is defined simply by multiplication by $\Omega \in U(\mathfrak{g}) \otimes U(\mathfrak{g})$. Now we note that $\Omega$ can be written as $\Delta(z)+z^{\prime} \otimes 1+1 \otimes z^{\prime \prime}$ for $z, z^{\prime}, z^{\prime \prime} \in Z(U(\mathfrak{g}))$. The naturality of $j$ implies at once that $j_{M, T_{1}}$ intertwines the endomorphisms arising by multiplication by $z^{\prime} \otimes 1$ and $1 \otimes z^{\prime \prime}$, so it remains to show that $j_{M, T_{1}}$ intertwines the endomorphisms arising by multiplication by $\Delta(z)$. This follows from Lemma 4.11(ii), establishing the claim.

Now to prove the theorem, we observe by Lemma 4.10 that $\mathrm{T}_{w_{0}}(M) \cong$ $P\left(A^{\Lambda}\right)$. Composing the isomorphism $j_{M, T_{d}}$ with any such isomorphism, we get 
a $\mathfrak{g}$-module isomorphism $\mathrm{T}_{w_{0}}\left(\widetilde{P}\left(A^{\Lambda}\right) \otimes T_{d}\right) \stackrel{\sim}{\rightarrow} P\left(A^{\Lambda}\right) \otimes T_{d}$ intertwining the two actions of $H_{d}$. This restricts to give the desired isomorphism $j_{\alpha}: \mathrm{T}_{w_{0}}\left(\widetilde{T}_{\alpha}^{\Lambda}\right) \stackrel{\sim}{\rightarrow}$ $T_{\alpha}^{\Lambda}$. Now consider the diagram from the statement of the lemma. In view of Lemma 4.10, the functor $T_{w_{0}}$ defines an isomorphism between $\operatorname{End}_{\mathfrak{g}}\left(\widetilde{T}_{\alpha}^{\Lambda}\right)^{\text {op }}$ and $\operatorname{End}_{\mathfrak{g}}\left(\mathrm{T}_{w_{0}}\left(\widetilde{T}_{\alpha}^{\Lambda}\right)\right)^{\mathrm{op}}$, which is why the bottom map in the diagram is an isomorphism. Now the fact that the diagram commutes follows because $j_{\alpha}$ intertwines the two actions of $H_{d}$. Finally, the last statement of the theorem follows directly from Theorem 3.6.

Theorem 4.13 (and Theorem [3.6) allows us henceforth to identify

$$
H_{\alpha}^{\Lambda}=\operatorname{End}_{\mathfrak{g}}\left(T_{\alpha}^{\Lambda}\right)^{\mathrm{op}}=\operatorname{End}_{\mathfrak{g}}\left(\widetilde{T}_{\alpha}^{\Lambda}\right)^{\mathrm{op}} .
$$

Recall now the Schur functor $\pi$ from (3.30). Analogously, we introduce the functor

$$
\widetilde{\pi}:=\operatorname{Hom}_{\mathfrak{g}}\left(\widetilde{T}_{\alpha}^{\Lambda}, ?\right): \widetilde{\mathcal{O}}_{\alpha}^{\Lambda} \rightarrow \operatorname{Rep}\left(H_{\alpha}^{\Lambda}\right)
$$

for any $\alpha \in Q_{+}$. The following lemma is the basic tool needed to deduce the main properties about this twisted Schur functor from the analogous properties involving $\pi$ from Theorem 3.7 .

Lemma 4.14. For $j_{\alpha}$ as in Theorem 4.13 and any $M \in \widetilde{\mathcal{O}}_{\alpha}^{\Lambda}$, there is a natural $H_{\alpha}^{\Lambda}$-module homomorphism $\gamma_{M}: \tilde{\pi}(M) \rightarrow \pi\left(\mathrm{T}_{w_{0}}(M)\right)$, such that $\gamma_{M}(f)=$ $\mathrm{T}_{w_{0}}(f) \circ j_{\alpha}^{-1}$ for each $f \in \tilde{\pi}(M)$. If $M$ has a standard filtration then $\gamma_{M}$ is an isomorphism.

Proof. Take $h \in H_{\alpha}^{\Lambda}$, and let $\theta_{h}$ (resp. $\widetilde{\theta}_{h}$ ) denote the endomorphism of $T_{\alpha}^{\Lambda}$ (resp. $\left.\widetilde{T}_{\alpha}^{\Lambda}\right)$ defined by right multiplication by $h$. For any $f \in \widetilde{\pi}(M)=\operatorname{Hom}_{\mathfrak{g}}\left(\widetilde{T}_{\alpha}^{\Lambda}, M\right)$, we have that $h f=f \circ \widetilde{\theta}_{h}$. Invoking Theorem 4.13 for the last equality, we get that

$$
\begin{aligned}
\gamma_{M}(h f) & =\gamma_{M}\left(f \circ \widetilde{\theta}_{h}\right)=\mathrm{T}_{w_{0}}\left(f \circ \widetilde{\theta}_{h}\right) \circ j_{\alpha}^{-1} \\
& =\mathrm{T}_{w_{0}}(f) \circ j_{\alpha}^{-1} \circ j_{\alpha} \circ \mathrm{T}_{w_{0}}\left(\widetilde{\theta}_{h}\right) \circ j_{\alpha}^{-1}=\gamma_{M}(f) \circ \theta_{h}=h \gamma_{M}(f) .
\end{aligned}
$$

This proves that $\gamma_{M}$ is an $H_{\alpha}^{\Lambda}$-module homomorphism, and it is clearly natural in $M$. Finally, suppose that $M$ has a standard filtration. As $\widetilde{T}_{\alpha}^{\Lambda}$ has a standard filtration too (e.g. by Lemma 4.12), Lemma 4.10 implies that the map

$$
\operatorname{Hom}_{\mathfrak{g}}\left(\widetilde{T}_{\alpha}^{\Lambda}, M\right) \rightarrow \operatorname{Hom}_{\mathfrak{g}}\left(\mathrm{T}_{w_{0}}\left(\widetilde{T}_{\alpha}^{\Lambda}\right), \mathrm{T}_{w_{0}}(M)\right)
$$

defined by applying the functor $\mathrm{T}_{w_{0}}$ is an isomorphism. Hence the map $\gamma_{M}$ is an isomorphism in this case.

The following theorem gives analogues of some parts of Theorem 3.7 in the twisted setup; all the other parts have obvious analogues too. Recall the definitions of $\widetilde{S}(A)$ and $\widetilde{D}(A)$ from (3.25) and (3.32).

Theorem 4.15. The following hold for $A \in \mathrm{Col}_{\alpha}^{\Lambda}$ :

(i) $\widetilde{\pi}(\widetilde{M}(A)) \cong \widetilde{S}(A) \cong S(A)^{\circledast}$;

(ii) $\widetilde{\pi}(\widetilde{L}(A))$ is non-zero if and only if $A \in \operatorname{Rev}_{\alpha}^{\Lambda}$, in which case we have that $\widetilde{\pi}(\widetilde{L}(A)) \cong \widetilde{D}(A) \cong D\left(A^{\downarrow}\right)$.

(iii) $\widetilde{\pi}(\widetilde{P}(A)) \cong \pi(T(A))$, which is isomorphic to $Y\left(A^{\downarrow}\right)$ in case $A \in \operatorname{Rev}_{\alpha}^{\Lambda}$. 
Proof. By Lemma 4.14, Lemma 4.10(i), Theorem 4.5, Theorem 3.7(iii) and (3.26), we have that

$$
\widetilde{\pi}(\widetilde{M}(A)) \cong \pi\left(\mathrm{T}_{w_{0}}(\widetilde{M}(A))\right) \cong \pi\left(M(A)^{\circledast}\right) \cong \pi(M(A))^{\circledast} \cong S(A)^{\circledast} \cong \widetilde{S}(A),
$$

giving (i). For (iii), we have by Lemma 4.14 and Lemma 4.10(ii) that

$$
\widetilde{\pi}(\widetilde{P}(A)) \cong \pi\left(\mathrm{T}_{w_{0}}(\widetilde{P}(A))\right) \cong \pi(T(A)) .
$$

Moreover if $A \in \operatorname{Rev}_{\alpha}^{\Lambda}$ then $T(A) \cong P\left(A^{\downarrow}\right)$ by Lemma 3.3, hence $\pi(T(A)) \cong$ $Y\left(A^{\downarrow}\right)$ by Theorem 3.7(ii). Finally to deduce (ii), note from Lemma 4.12 that $\widetilde{\pi}(\widetilde{L}(A))$ is non-zero if and only if $A \in \operatorname{Rev}_{\alpha}^{\Lambda}$. Moreover, assuming $A \in \operatorname{Rev}_{\alpha}^{\Lambda}$, we get that $\widetilde{\pi}(\widetilde{L}(A))$ is an irreducible $H_{\alpha}^{\Lambda}$-module exactly as in Theorem 3.7(i), and $\widetilde{\pi}(\widetilde{P}(A)) \cong Y\left(A^{\downarrow}\right)$ is its projective cover exactly as in Theorem 3.7(ii). Since $Y\left(A^{\downarrow}\right)$ is the projective cover of $D\left(A^{\downarrow}\right)$, we deduce that $\widetilde{\pi}(\widetilde{L}(A)) \cong D\left(A^{\downarrow}\right)$, which is isomorphic to $\widetilde{D}(A)$ by (3.32).

4.4. Signed Young modules. For completeness, we want finally to explain briefly how to identify the modules in Theorem 4.15)(iii) with signed Young modules. We omit some of the details in the proofs here. Assuming to start with that $I$ is arbitrary, take any $A \in \mathrm{Col}_{\alpha}^{\Lambda}$, and let $\widetilde{X}(A)$ denote the left ideal of $H_{\alpha}^{\Lambda}$ generated by the element

$$
\prod_{i=1}^{l-1} \prod_{j=1}^{d_{i+1}+\cdots+d_{l}}\left(x_{j}-m_{i}\right) \cdot \sum_{w \in S_{(\lambda(l))^{t}} \times \cdots \times S_{\left(\lambda^{(1)}\right)^{t}}} \operatorname{sgn}(w) w,
$$

where $\lambda=\left(\lambda^{(1)}, \ldots, \lambda^{(l)}\right):=\lambda(A)$ and $d_{i}:=\left|\lambda^{(i)}\right|$. This is the reduced signed permutation module as in [M, §4]. The next lemma explains how to define the signed Young module $\widetilde{Y}(A)$ as a summand of $\widetilde{X}(A)$.

Lemma 4.16. There exists a unique (up to isomorphism) indecomposable $H_{\alpha}^{\Lambda}-$ module $\widetilde{Y}(A)$ such that

$$
\widetilde{X}(A)=\tilde{Y}(A)+(\ddagger)
$$

where $(\ddagger)$ denotes a direct sum of $\tilde{Y}(B)$ 's for $B \in \mathrm{Col}_{\alpha}^{\Lambda}$ with $\lambda(B)<\lambda(A)$ in the dominance ordering on multipartitions.

Proof. We may assume for the proof that $I=\mathbb{Z}$, so that the automorphism $\sigma$ from (3.39) makes sense. By (4.3) and (4.12), we have that $\widetilde{X}(A)=\sigma^{*}\left(X\left(A^{t}\right)\right)$. Setting

$$
\widetilde{Y}(A):=\sigma^{*}\left(Y\left(A^{t}\right)\right)
$$

and noting that the bijection (3.40) is order-reversing with respect to the dominance ordering, the lemma now follows from the first part of Theorem 4.6.

For the remainder of the subsection we assume that $I$ is bounded-below. In the same spirit as (4.4), we define the exterior power module

$$
E(A):=U(\mathfrak{g}) \otimes_{U(\mathfrak{p})}\left[I^{\Lambda} \otimes \bigwedge^{\left(\lambda^{(l)}\right)^{t}}\left(V^{l}\right) \otimes \cdots \otimes \bigwedge^{\left(\lambda^{(1)}\right)^{t}}\left(V^{1}\right)\right] .
$$

Here, $V^{i}$ denotes the quotient of the natural module of column vectors by the p-submodule $V_{i-1}$ defined just after (4.4). Also for a vector space $E$ and a partition $\mu$ of $n$ we write $\bigwedge^{\mu}(E)$ for the quotient $\bigwedge^{\mu_{1}}(E) \otimes \bigwedge^{\mu_{2}}(E) \otimes \cdots$ of 
$E^{\otimes n}$. By arguments similar to the proof of (4.5) (which is BK3, Theorem 4.14]), one shows that

$$
E(A)=T(A)+(\#)
$$

where (\#) is a direct sum of $T(B)$ 's for $B \in \mathrm{Col}^{\Lambda}$ with $\lambda(B)<\lambda(A)$ in the dominance order; the key point is that $R\left(T\left(A^{\prime}\right) \otimes T\left(A^{\prime \prime}\right)\right) \cong T(A)$ in the setup and notation of [BK3, Corollary 4.12(i)]. Moreover it is the case that

$$
\widetilde{X}(A) \cong \operatorname{Hom}_{\mathfrak{g}}\left(T_{\alpha}^{\Lambda}, E(A)\right) .
$$

This is proved by the same techniques used to prove (4.6) (which is BK3, Theorem 6.9]); in particular the proof goes via finite $W$-algebras. (There are some additional complications in the present setting since this argument realizes $\pi(T(A))$ initially as a quotient of $H_{\alpha}^{\Lambda}$, whereas $\widetilde{Y}(A)$ is a submodule; this is overcome by using Theorem 4.5, the self-duality of the tilting module $E(A)$, and the symmetric algebra structure on $H_{\alpha}^{\Lambda}$.)

Theorem 4.17. $\pi(T(A)) \cong \widetilde{Y}(A)$.

Proof. This follows from (4.15), Lemma 4.16, Theorem 3.7(vi) and (4.16), in exactly the same way that Theorem 4.6(ii) was deduced from (4.5) in the proof of that theorem.

We say that $\tilde{Y}$ is a signed Young generator for $H_{\alpha}^{\Lambda}$ if it is a finite direct sum of the signed Young modules $\tilde{Y}(A)$ for $A \in \mathrm{Col}_{\alpha}^{\Lambda}$ with each appearing at least once.

Corollary 4.18. If $\tilde{Y}$ is a signed Young generator for $H_{\alpha}^{\Lambda}$ then the category of finite dimensional left modules over the algebra $\operatorname{End}_{H_{\alpha}^{\Lambda}}(\widetilde{Y})^{\mathrm{op}}$ is equivalent to the category $\widetilde{O}_{\alpha}^{\Lambda}$. Moreover $\operatorname{End}_{H_{\alpha}^{\Lambda}}(\widetilde{Y})^{\mathrm{op}}$ is the Ringel dual of the algebra

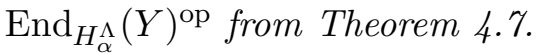

Proof. By Theorems 4.17 and 4.15)(iii), there is a tilting generator $T$ for $\mathcal{O}_{\alpha}^{\Lambda}$ and a projective generator $\widetilde{P}$ for $\widetilde{\mathcal{O}}_{\alpha}^{\Lambda}$ such that $\pi(T) \cong \widetilde{Y} \cong \widetilde{\pi}(\widetilde{P})$. By Theorem 3.7(vi) and Lemma 4.10, we get that

$$
\operatorname{End}_{\mathfrak{g}}(T)^{\mathrm{op}} \cong \operatorname{End}_{H_{\alpha}^{\Lambda}}(\widetilde{Y})^{\mathrm{op}} \cong \operatorname{End}_{\mathfrak{g}}(\widetilde{P})^{\mathrm{op}} .
$$

Recalling Theorem 4.7, the left hand algebra is the Ringel dual of the algebra $\operatorname{End}_{H_{\alpha}^{\Lambda}}(Y)^{\mathrm{op}}$, while the category of finite dimensional modules over the right hand algebra is obviously equivalent to $\widetilde{\mathcal{O}}_{\alpha}^{\Lambda}$.

Corollary 4.19. The following hold for any $A \in \mathrm{Col}_{\alpha}^{\Lambda}$ :

(i) $Y(A)^{\circledast} \cong Y(A)$;

(ii) $\tilde{Y}(A)^{\circledast} \cong \widetilde{Y}(A)$.

Proof. We get that $\tilde{Y}(A)^{\circledast} \cong \widetilde{Y}(A)$ immediately from Theorem 4.17, as the tilting module $T(A)$ is self-dual and the Schur functor $\pi$ commutes with duality according to Theorem 4.5. This proves (ii). Then (i) follows from (ii) by twisting with the automorphism $\sigma$, using (3.36) and (4.13). 


\section{REFERENCES}

[AnS] H. Andersen and C. Stroppel, Twisting functors on $\mathcal{O}$, Represent. Theory 7 (2003), 681-699.

[AS] T. Arakawa and T. Suzuki, Duality between $\mathfrak{s l}_{n}(\mathbb{C})$ and the degenerate affine Hecke algebra, J. Algebra 209 (1998), 288-304.

[A1] S. Ariki, On the decomposition numbers of the Hecke algebra of $G(m, 1, n), J$. Math. Kyoto Univ. 36 (1996), 789-808.

[A2] S. Ariki, Proof of the modular branching rule for cyclotomic Hecke algebras, J. Algebra 306 (2006), 290-300.

[AMR] S. Ariki, A. Mathas and H. Rui, Cyclotomic Nazarov-Wenzl algebras, Nagoya Math. J. 182 (2006), 47-134.

[B] E. Backelin, Koszul duality for parabolic and singular category $\mathcal{O}$, Represent. Theory 3 (1999), 139-152.

[BB] A. Beilinson and J. Bernstein, Localisation de g-modules, C. R. Acad. Sci. Paris Ser. I Math. 292 (1981), 15-18.

[BGS] A. Beilinson, V. Ginzburg and W. Soergel, Koszul duality patterns in representation theory, J. Amer. Math. Soc. 9 (1996), 473-527.

[BFK] J. Bernstein, I. Frenkel and M. Khovanov, A categorification of the Temperley-Lieb algebra and Schur quotients of $U\left(\mathfrak{s l}_{2}\right)$ via projective and Zuckerman functors. Selecta Math. (N.S.) 5 (1999), 199-241.

[BGG] J. Bernstein, I. M. Gelfand and S. I. Gelfand, A category of g-modules, Func. Anal. Appl. 10 (1976), 87-92.

[B1] J. Brundan, Dual canonical bases and Kazhdan-Lusztig polynomials, J. Algebra 306 (2006), 17-46.

[B2] J. Brundan, Centers of degenerate cyclotomic Hecke algebras and parabolic category $\mathcal{O}$, Represent. Theory 12 (2008), 236-259.

[BDK] J. Brundan, R. Dipper and A. Kleshchev, Quantum linear groups and representations of $G L_{n}\left(\mathbb{F}_{q}\right)$, Mem. Amer. Math. Soc. 149 (2001), no. 706, 112 pages.

[BK1] J. Brundan and A. Kleshchev, Translation functors for general linear and symmetric groups, Proc. London Math. Soc. 80 (2000), 75-106.

[BK2] J. Brundan and A. Kleshchev, Representations of shifted Yangians and finite $W$ algebras, Mem. Amer. Math. Soc. 196 (2008), no. 918, 107 pages.

[BK3] J. Brundan and A. Kleshchev, Schur-Weyl duality for higher levels, Selecta Math. (N.S.) 14 (2008), 1-57.

[BK4] J. Brundan and A. Kleshchev, Blocks of cyclotomic Hecke algebras and KhovanovLauda algebras; arXiv:0808.2032

[BS] J. Brundan and C. Stroppel, Highest weight categories arising from Khovanov's diagram algebra III: category $\mathcal{O}$; arXiv:0812.1090

[BrK] J.-L. Brylinksi and M. Kashiwara, Kazhdan-Lusztig conjecture and holonomic systems, Invent. Math. 64 (1981), 387-410.

[CG] N. Chriss and V. Ginzburg, Representation Theory and Complex Geometry, Birkhäuser, 1997.

[CR] J. Chuang and R. Rouquier, Derived equivalences for symmetric groups and $\mathfrak{s l}_{2}-$ categorification, Ann. of Math. 167 (2008), 245-298.

[DJM] R. Dipper, G. James and A. Mathas, Cyclotomic q-Schur algebras, Math. Z. 229 (1999), 385-416.

[D] V. Drinfeld, Degenerate affine Hecke algebras and Yangians, Func. Anal. Appl. 20 (1986), 56-58.

[F] W. Fulton, Young Tableaux, London Math. Soc., 1997.

[G] P. Gabriel, Des catégories Abéliennes, Bull. Soc. Math. France 90 (1962), 323-448.

[G1] I. Grojnowski, Representations of affine Hecke algebras (and affine quantum $\mathrm{GL}_{n}$ ) at roots of unity, Internat. Math. Res. Not. 5 (1994), 215-217.

[G2] I. Grojnowski, Affine $\widehat{\mathfrak{s l}}_{p}$ controls the modular representation theory of the symmetric group and related Hecke algebras; math.RT/9907129v1. 
[H] A. Henderson, Nilpotent orbits of linear and cyclic quivers and Kazhdan-Lusztig polynomials of type A, Represent. Theory 11 (2007), 95-121.

[J] N. Jacon, On the parametrization of the simple modules for Ariki-Koike algebras at roots of unity, J. Math. Kyoto Univ. 44 (2004), 729-767.

[K1] M. Kashiwara, Global crystal bases of quantum groups, Duke Math. J. 69 (1993), 455-485.

[K2] M. Kashiwara, On crystal bases, in: "Representations of Groups," CMS Conf. Proc. 16, Amer. Math. Soc., 1995, pp. 155-197.

[KN] M. Kashiwara and T. Nakashima, Crystal graphs for representations of the $q$-analogue of classical Lie algebras, J. Algebra 165 (1994), 295-345.

[KL1] D. Kazhdan and G. Lusztig, Representations of Coxeter groups and Hecke algebras, Invent. Math. 53 (1979), 165-184.

[KL2] D. Kazhdan and G. Lusztig, Proof of the Deligne-Langlands conjecture for Hecke algebras, Invent. Math. 87 (1987), 153-215.

[K] A. Kleshchev, Linear and Projective Representations of Symmetric Groups, Cambridge University Press, Cambridge, 2005.

[LLT] A. Lascoux, B. Leclerc and J.-Y. Thibon, Hecke algebras at roots of unity and crystal bases of quantum affine algebras, Comm. Math. Phys. 181 (1996), 205-263.

[LS] A. Lascoux and M.-P. Schützenberger, Keys and standard bases, in: "Invariant Theory and Tableaux", D. Stanton ed., Springer 1990.

[LT] B. Leclerc and J.-Y. Thibon, Canonical bases of $q$-deformed Fock spaces, Internat. Math. Res. Notices 9 (1996), 447-456.

[L1] G. Lusztig, Cuspidal local systems and graded Hecke algebras I, Inst. Hautes Études Sci. Publ. Math. 67 (1988), 145-202.

[L2] G. Lusztig, Quantum groups at roots of 1, Geom. Ded. 35 (1990), 89-114.

[L3] G. Lusztig, Quivers, perverse sheaves, and quantized enveloping algebras, J. Amer. Math. Soc. 4 (1991), 365-421.

[L4] G. Lusztig, Introduction to Quantum Groups, Birkhäuser, 1993.

[L5] G. Lusztig, Cuspidal local systems and graded Hecke algebras, II, in: "Representations of Groups," CMS Conf. Proc. 16, Amer. Math. Soc., 1995, pp. 217-275.

[M] A. Mathas, Tilting modules for cyclotomic Schur algebras, J. reine angew. Math. 562 (2003), 137-169.

[R] J. Rickard, Equivalences of derived categories for symmetric algebras, J. Algebra 257 (2002), 460-481.

[S] W. Soergel, Character formulas for tilting modules over Kac-Moody algebras, Represent. Theory 2 (1998), 432-448.

[VV] M. Varagnolo and E. Vasserot, On the decomposition matrices of the quantized Schur algebra, Duke Math. J. 100 (1999), 267-297.

[Z1] A. Zelevinsky, p-adic analogue of the Kazhdan-Lusztig hypothesis, Funct. Anal. Appl. 15 (1981), 83-92.

[Z2] A. Zelevinsky, Two remarks on graded nilpotent orbits, Russian Math. Surveys 40 (1985), 249-250.

Department of Mathematics, University of Oregon, Eugene OR 97403, USA

E-mail address: brundan@uoregon.edu

Department of Mathematics, University of Oregon, Eugene OR 97403, USA

E-mail address: klesh@uoregon.edu 